\title{
Spin rates of V-type asteroids
}

\author{
Dagmara Oszkiewicz ${ }^{1}$, Volodymyr Troianskyi ${ }^{1,2}$, Dóra Föhring ${ }^{3}$, Adrián Galád ${ }^{4}$, Tomasz Kwiatkowski ${ }^{1}$, \\ Anna Marciniak ${ }^{1}$, Brian A. Skiff ${ }^{5}$, Stefan Geier ${ }^{6,7}$, Wojciech Borczyk ${ }^{1, \dagger}$, Nicholas A. Moskovitz ${ }^{5}$, \\ Paweł Kankiewicz ${ }^{8}$, Štefan Gajdoš ${ }^{4}$, Jozef Világi ${ }^{4}$, L’udovít Polčic ${ }^{4}$, Tomasz Kluwak ${ }^{9}$, Emil Wilawer ${ }^{1}$, \\ Volodymyr Kashuba ${ }^{2}$, Sergei Udovichenko ${ }^{2}$, Leonid Keir ${ }^{2}$, Krzysztof Kamiński ${ }^{1}$, \\ Maxime Devogele ${ }^{5}$, and Annika Gustafsson ${ }^{5,10}$
}

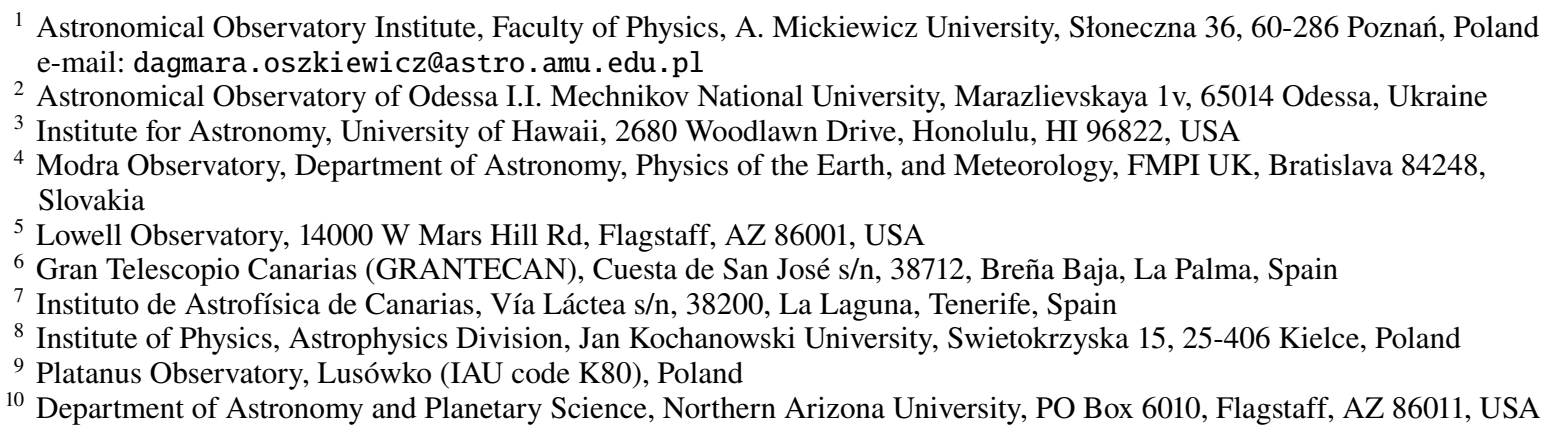

Received 1 April 2020 / Accepted 29 September 2020

\begin{abstract}
Context. Basaltic V-type asteroids play a crucial role in studies of Solar System evolution and planetesimal formation. Comprehensive studies of their physical, dynamical, and statistical properties provide insight into these processes. Thanks to wide surveys, currently there are numerous known V-type and putative V-type asteroids, allowing a detailed statistical analysis.

Aims. Our main goal is to analyze I corrected for US language conventions in this paper the currently available large sample of V-type spin rates, to find signatures of the non-gravitational Yarkovsky-O'Keefe-Radzievskii-Paddack (YORP) effect among the different V-type populations, and to estimate the spin barrier and critical density for V-type asteroids. Our intention is to increase the pool of information about the intriguing V-types.

Methods. We collected rotational periods from the literature for spectrally confirmed V-types, putative V-types, and Vesta family members. Through spectroscopic observations we confirmed their taxonomic type and verified the high confirmation rates of the putative V-types. We combined the collected periods with periods estimated in this manuscript and produced rotational frequency distributions. We determined the spin barrier in the frequency-light curve amplitude space for V-type asteroids.

Results. We analyzed rotational periods of 536 asteroids in our sample. As expected, due to the small size of the objects analyzed, the frequency distributions for the Vesta family and the V-types outside the family are inconsistent with a Maxwellian shape. The Vesta family shows an excess of slow-rotators. V-types outside the family show an excess of both slow and fast rotators. Interestingly, we found that the population of V-types outside the Vesta family shows a significant excess of fast rotators compared to the Vesta family. The estimated critical density for V-type asteroids exceeds $\rho_{\mathrm{c}}=2.0 \mathrm{~g} \mathrm{~cm}^{-3}$, which surpasses the previous estimates.

Conclusions. We demonstrated that V-type asteroids have been influenced by the thermal radiation YORP effect and that their critical spin rate is higher than for C-type asteroids. The population of V-types outside the Vesta family shows a significant excess of fast rotators compared to the Vesta family. We hypothesize that the objects that evolved from the Vesta family though the Yarkovsky drift are also more susceptible to the YORP effect. Objects for which YORP has not yet had enough time to act and those that are more YORP resistant will be left in the family, which explains the relatively small proportion of fast rotators being left. The YORP timescale must thus be similar to the migration timescale for those objects.
\end{abstract}

Key words. minor planets, asteroids: general

\section{Introduction}

Basaltic V-type asteroids and their fragments, howardite-eucritediogenite (HED) meteorites, have been studied for about half a century. They provide important clues to the differentiation process, constrain the spatial mineralogical distribution and temperature gradient of the early Solar nebula, and even reveal some of the dynamical processes in the Solar System.

Asteroid (4) Vesta was the first object to be identified as basaltic (McCord et al. 1970; Consolmagno \& Drake 1977).

\footnotetext{
${ }^{\dagger}$ Deceased.
}

A number of spectroscopically similar asteroids in the vicinity of (4) Vesta, some of which were not Vesta family members, were later identified (Binzel \& Xu 1993; Florczak et al. 2002; Lazzaro et al. 2004; Alvarez-Candal et al. 2006; Burbine et al. 2001). Numerical studies showed that those outside the Vesta family borders might have escaped through a combination of the Yarkovsky effect and dynamical resonances (Carruba et al. 2005; Nesvorný et al. 2008). Furthermore, two large collisional events are believed to have produced Vestoids (Milani et al. 2014; Spoto et al. 2015); two large craters found on the surface of Vesta are thought to be evidence of this (Thomas et al. 1997; Schenk et al. 2012). The surface of these craters was mapped by the Dawn 
mission (Roatsch et al. 2012) and provided further mineralogical evidence of the Vesta-V-type asteroid-HED meteorite link (De Sanctis et al. 2013, 2012; Russell et al. 2012; McSween et al. 2013).

Some V-type asteroids are well separated from Vesta and are thus considered non-Vestoids (Lazzaro et al. 2000; Roig et al. 2008; Moskovitz et al. 2008a; Duffard \& Roig 2009; Ieva et al. 2015, 2018; Migliorini et al. 2017). These asteroids show spectral properties distinct from Vestoids (Hardersen et al. 2004a; Hammergren et al. 2006; Leith et al. 2017; Ieva et al. 2015, 2018; Medeiros et al. 2019) and have a low probability ( $1 \%)$ of having evolved from Vesta (Roig et al. 2008; Carruba et al. 2007; Michtchenko et al. 2002). Carruba et al. (2014) indicated two possible source regions for V-types in the middle main belt, and Huaman et al. (2014) three of these in the outer main belt.

There is some debate about the presence of non-Vestoids in the inner main belt (Bottke et al. 2006a; Nesvorný et al. 2008; Scott et al. 2009; Roig et al. 2008; Moskovitz et al. 2010; Wasson 2013). Particularly, the low inclination $\left(i \leq 6^{\circ}\right)$ V-types in the inner main belt were not sufficiently reproduced in numerical simulations by Nesvorný et al. (2008) and may originate in either a distinct parent body or from an older impact on Vesta. Furthermore, some V-types in the inner main belt have rotational properties inconsistent with migration from Vesta (Oszkiewicz et al. 2015, 2017).

This complex population is spread over the entire main belt. The V-type population is predominantly composed of small objects $(<10 \mathrm{~km}$ in diameter), well within the range in which the Yarkovsky-O'Keefe-Radzievskii-Paddack (YORP) effect is expected to be significant. Distribution of rotational frequencies of the V-type asteroids has been so far poorly understood due to the limited number of objects studied (i.e., Hasegawa et al. 2014 examined the frequency distribution of 59 V-type asteroids). However, due to large surveys such as the SDSS and HST-VISTA, a large number of putative V-types can now be added to the spectrally known V-types and Vesta family members resulting in a large enough sample ( $>500$ objects) for a statistical YORP analysis.

The YORP effect (Bottke et al. 2006b) modifies the Maxwellian rotational-frequency distribution expected from a purely collisionally evolved population (Salo 1987). This effect spins up or down irregularly shaped asteroids (Rubincam 2000) creating an excess of slow and fast rotators, thereby diluting the rotation spin distributions from Maxwellian. Large asteroids ( $>40 \mathrm{~km}$ in diameter) could retain primordial spins, whereas smaller objects are more affected by the YORP effect (Pravec et al. 2002). An excess of fast to slow rotators has been previously observed for the Flora, Hungaria, and Koronis families (Warner et al. 2009a; Kryszczyńska et al. 2012; Slivan et al. 2003, 2009) and generally for the population of small main belt (MBA) and Mars-crossing asteroids (Pravec et al. 2008). Direct detections of the YORP effect spinning up objects have been made for few asteroids so far (Lowry et al. 2007; Durech et al. 2008; Kaasalainen \& Durech 2007; Taylor et al. 2007).

The YORP effect may also spin up objects over the spin barrier causing their disintegration and/or the formation of binary asteroids (Walsh et al. 2008). The spin barrier has been estimated at $\mathrm{P}$ about $2.2 \mathrm{~h}$ (or equivalently rotational frequency $\omega$ of around $\left.10 \mathrm{~d}^{-1}\right)$ for the general population of small $(10 \mathrm{~km}>D>150 \mathrm{~m})$ asteroids (Harris 1996). Rubble-pile asteroids with a lower bulk density are expected to have a lower spin rate limit (Pravec \& Harris 2000), thus the overall spin rate of C-type asteroids should be lower than that of the V- and S-type objects. Chang et al. (2015) estimated the critical density for S- and V-type asteroids independently as $\rho=2.0 \mathrm{~g} \mathrm{~cm}^{-3}$, and for C-type objects as $\rho=$ $1.5 \mathrm{~g} \mathrm{~cm}^{-3}$. In that work it was also suggested that there is a need to confirm the results after supplementing the sample of objects. We are now dealing with a statistically larger sample because we supported the calculations with a significant number of new observations and putative V-types, described in the next section.

In Sect. 2, we describe the spectroscopic and photometric observations and the data reduction process. In Sect. 3, we taxonomically classify the observed asteroids and verify the high confirmation rate of putative V-types. New rotational periods are determined in Sect. 3. In Sect. 4, we perform statistical spin rate analysis for various V-type populations. The summary of our results is presented in Sect. 5 .

\section{Observations}

\subsection{Spectroscopic observations and data reduction}

We obtained visible (VIS) spectra of 17 V-type and V-type candidate asteroids at three telescopes. The V-type candidates were selected out of putative V-types listed in the literature (Roig \& Gil-Hutton 2006; Carvano et al. 2010; Hasselmann et al. 2012; Licandro et al. 2017; Moskovitz et al. 2008b). Most of the data were taken at the $2.5 \mathrm{~m}$ Nordic Optical Telescope (NOT), spectra of two asteroids were obtained at the $10 \mathrm{~m}$ South African Large Telescope (SALT) and the $4.3 \mathrm{~m}$ Lowell Discovery Telescope (LDT). The observing circumstances and optical elements used are summarized in Table A.1.

At the NOT we used the Alhambra Faint Object Spectrograph and Camera (ALFOSC) combined with grism NOT-11 or grism NOT-12 depending on the night. Both grisms are low resolution. Grism 11 covers wavelengths 3900-10000 A; however, fringes are noticeable starting from around $7500 \AA$. Grism 12 covers wavelengths 5100-11 $000 \AA$, with fringes starting also around $7500 \AA$. It should be noted that grism 12 has deteriorated, and thus higher order Legendre polynomials had to be used to extract the spectra, and lamp identification was challenging. For this reason we repeated observations of some of the targeted asteroids with grism 11. For most objects we found that grism 12 is still usable; however, the reduction process was cumbersome and problematic, and the results questionable for some objects. Thus, we would recommend the decommissioning of grism 12 . In Table A.1 we list the observed targets, telescope, and setup used. Slit widths are adjusted to seeing. We used non-sidereal tracking together with a five-point dither to obtain several spectra. The spectra were corrected for flat and bias. Wavelength calibration was done using $\mathrm{He}, \mathrm{Ne}$, and ThAr lamps. The collected spectra were averaged and divided by solar analog and normalized at 0.55 microns.

At the SALT we used the Robert Stobie Spectrograph (RSS) together with the low resolution grism PG0300 covering wavelengths 3700-9000 $\AA$. Our reduction procedure followed that described in Oszkiewicz et al. (2014).

\subsection{Photometric observations and data reduction}

Photometric observations were conducted using several telescopes listed in Table 1 . Most of the data were taken with a Cousins $R_{\mathrm{C}}$ filter, but some with Johnson $V$, wide-band VR, or a clear filter. The VR filter used at the Hall telescope at Lowell Observatory has a passband similar to Sloan $r^{\prime}$. The half maximum wavelengths are close to 5200 and $7000 \AA$, compared to the Sloan $r^{\prime}$ limits of about 5600 and $6950 \AA$. Thus, it is only 
Table 1. Telescopes and instruments used for the photometric observations.

\begin{tabular}{lcccccc}
\hline \hline Telescope & Observatory & Instrument & Aperture & IAU code & Bands & No. of nights \\
\hline Perkins & Lowell Observatory & Prism & $1.83 \mathrm{~m}$ & 688 & $R$ & 2 \\
Hall & Lowell Observatory & Nasa42 & $1.07 \mathrm{~m}$ & 688 & $R, V R$ & 20 \\
31 inch & Lowell Observatory & Nasa31 & $0.79 \mathrm{~m}$ & 688 & $R$ & 6 \\
UH88 & Mauna Kea-UH & Tektronix $2048^{2} \mathrm{CCD}$ & $2.24 \mathrm{~m}$ & $\mathrm{~T} 12$ & $r^{\prime}$ & 12 \\
JKT & La Palma & Andor CCD & $1.0 \mathrm{~m}$ & 950 & $R, V$ & 13 \\
RBT & Winer Observatory & Andor iXon 888 & $0.7 \mathrm{~m}$ & 648 & $R, L$ & 6 \\
Zeiss & Modra Observatory & Apogee Ap8p & $0.6 \mathrm{~m}$ & 118 & $R$ & 144 \\
OMT-800 & Odessa-Mayaki & FLI 9000 & $0.8 \mathrm{~m}$ & 583 & $V$ & 5 \\
AZT-3 & Odessa-Mayaki & UAI CCD & $0.48 \mathrm{~m}$ & 583 & $R$ & 5 \\
Total & & & & & 213 \\
\hline
\end{tabular}

slightly wider on the blue side of the nominal $\mathrm{r}^{\prime}$ passband. The $L$ filter used at the RBT telescope at Winer Observatory is an interferometric luminosity filter with a transmission window from 3700 to $7000 \AA$. Altogether we obtained data on 213 nights, mostly at Modra Observatory and Lowell Observatory. We performed standard photometric reduction with bias and flat-field correction followed by ordinary aperture photometry. We used the commercial software Canopus (version 10.7.12.7) for data from the Lowell Observatory, Mauna Kea Observatories, and Odessa-Mayaki Observatory, making use of its built-in photometric catalog and Gaia DR2 catalog to obtain approximate $R_{\mathrm{C}}$ or Sloan $r^{\prime}$ nightly zero points. At Modra Observatory a clear filter was used, and for the data processing MaxIm DL and their own software. Linkages to nearly the same magnitude level (within $0.05 \mathrm{mag}$ ) were done using the Carlsberg Meridian Catalogue stars with the help of Astrometrica (method described in Dymock \& Miles 2009). This resulted in magnitudes close to the Cousins $R$ filter. We also used the Starlink package (version NAMAKA) for data from the Roque de los Muchachos Observatory, Kitt Peak Observatory, and Winer Observatory. We generally used three (in Starlink) to five (in Canopus) comparison stars in each field, usually of much higher signal-to-noise ratio than the target, and selected to have nearasteroidal colors if possible (i.e., roughly $0.5<B-V<0.95$ or $0.35<g-r<0.85$ ). For asteroids near their stationary points, we could use the same comparison star sets on multiple nights. The measuring apertures were generally $8-12$ arcsec in diameter, depending on the image quality. The typical mean nightly root mean square scatter of the ensemble of comparison stars was 0.006-0.01 mag.

\section{Results}

\subsection{Spectral classification}

We report spectra of 17 asteroids, for 11 of which optical spectra were obtained for the first time. Out of these 7 asteroids had not been observed at either near-infrared (NIR) or visible and infrared (VIR) wavelengths previously, thus those constitute new classifications. Asteroids (1914) Hartbeespoortdam, (2704) Julian Loewe, (4434) Nikulin, (5037) Habing, 5525 (1991 TS4), and (7558) Yurlov were observed for comparison with the literature (Alvarez-Candal et al. 2006; Spahr et al. 1997; Burbine \& Binzel 2002; Bus \& Binzel 2002; Solontoi et al. 2012; Wisniewski 1991; Moskovitz et al. 2008b). In Fig. 1, we present all the obtained optical spectra, and in Fig. 2, the spectra concatenated with available NIR spectra from the literature. The obtained spectra closely match those reported in the literature.

We classified the observed spectra using the M4AST online tool and the standard error method of Popescu et al. (2012). In Table 2 we report two closest classes for each spectrum, along with the distance to the class templates and reliability as defined by Popescu et al. $(2012,2011)$. Reliability is a function of wavelength range covered and number of discrete data points used in the classification. The mathematical formulation of this parameter can be found in Popescu et al. (2012, 2011). Most of our targets (with the exception of 3302 Schliemann and 96521996 AF2) are consistent with the V- or R-type templates (DeMeo et al. 2009). The computed distance between the data and the $\mathrm{V}$ - and R-templates is very similar. This is due to the fact that the two classes are very similar in VIS wavelengths and differ more in the NIR wavelengths. Since R-types are very rare we consider the 15 observed objects V-types. This results in an $88 \%$ rate of success for confirmation on V-types.

High confirmation rates were also found by other authors i.e., $90 \%$ in Moskovitz et al. (2008b) or 78\% in Solontoi et al. (2012). This very high confirmation rate leads us to assume that most of the asteroids that have their SDSS colors consistent with the V-type template are indeed V-types, and thus may be used in further analysis.

In Table 3 we derived the reflectivity gradient in the 5000 $7500 \AA$ and 8000-9200 $\AA$ range (slope A and slope B, respectively) and $1.0 \mu \mathrm{m}$ band depth (as in Ieva et al. 2015) for the observed asteroids. As noted by Ieva et al. (2015) a steeper slope A could be indicative of weathered surfaces (Fulvio et al. 2012, 2016), while a deeper apparent depth could be indicative of bigger grain size or fresh unweathered pyroxene (Hiroi et al. 1995; Cloutis et al. 2013). The derived parameters are consistent with typical Vestoidal values. We do not list parameters for objects observed with grism 12 because the mechanical damage of the grism the wavelength calibration required fitting a high order polynomial and may be imprecise. Some spectra covered the $1.0 \mu \mathrm{m}$ band well, which allowed us to determine band minima (BI) by fitting a third-degree polynomial. The resulting values are listed in Table 3.

For asteroid 8645 we obtained both VIS and NIR spectra for the first time. For asteroids (5235) Jean-Loup and (5560) Amytis the obtained spectra are complementary to the NIR data in the literature. The minima of the $1.0 \mu \mathrm{m}$ band that we obtained are consistent, within the uncertainties, with those derived by Hardersen et al. (2014b) based on NIR spectra. We note that the variability of methods used for band characterization between 

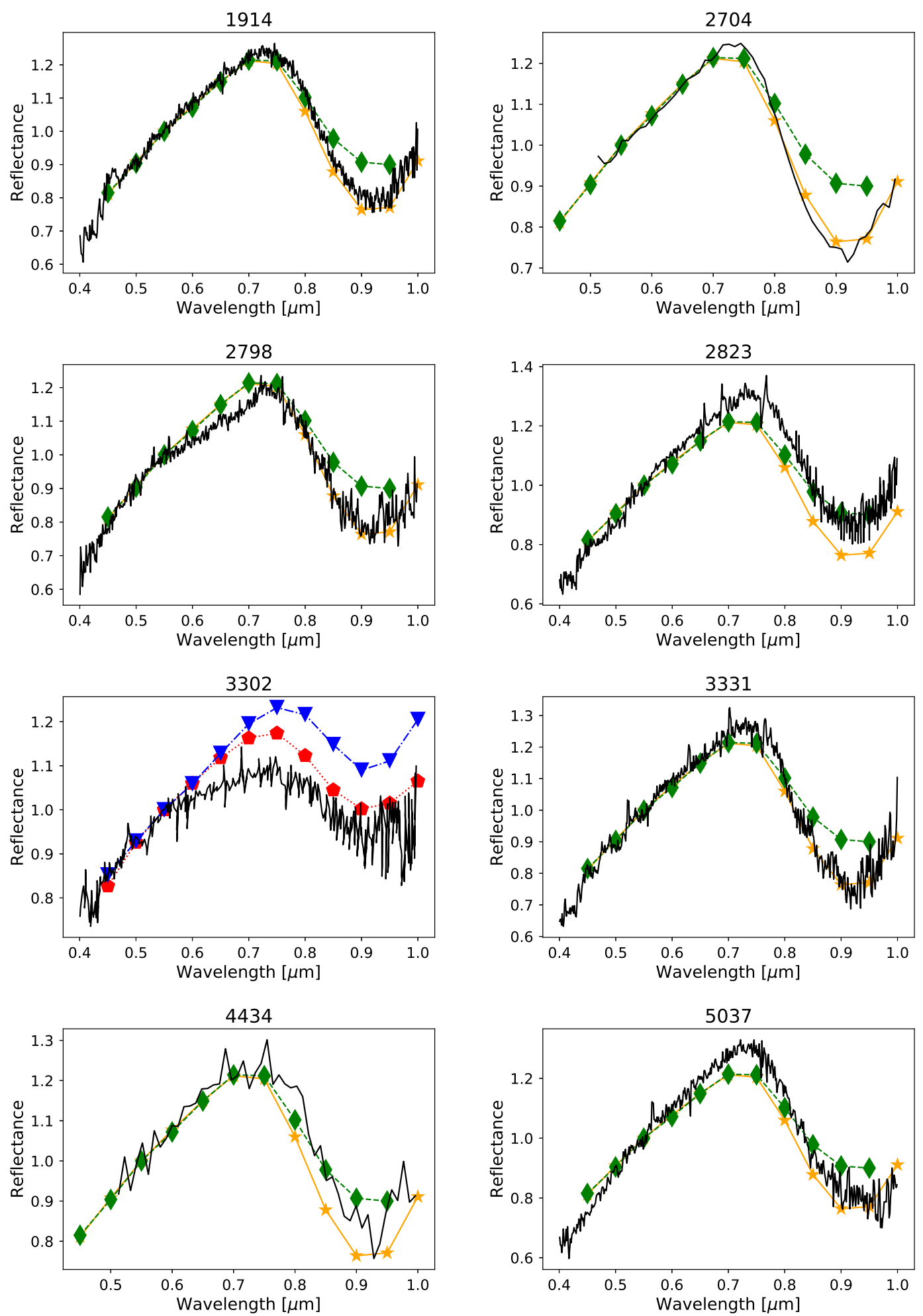

Fig. 1. Visible spectra obtained in this project. Spectral templates are denoted as follows: V (orange stars), R (green diamonds), Sr (red pentagons), Sv (blue triangles).

different authors does not allow a direct comparison of the spectral parameters. For example, Moskovitz et al. (2010) used polynomial fits and continuum removal for the $1.0 \mu \mathrm{m}$ band, but not for the $2.0 \mu \mathrm{m}$ band; Leith et al. (2017) used a modified Gaussian model (Sunshine et al. 1990); and Birlan et al. (2016) followed the method of Cloutis et al. (1986), which uses polynomial fitting in selected regions for finding band minima and removing of double sloped linear continuum for the band centers.

\subsection{Rotational periods outside the Vesta family asteroids}

We determined new rotational periods for $18 \mathrm{~V}$-type asteroids outside the dynamical Vesta family. We list them in Table 4 along 
D. Oszkiewicz et al.: Spin rates of V-types
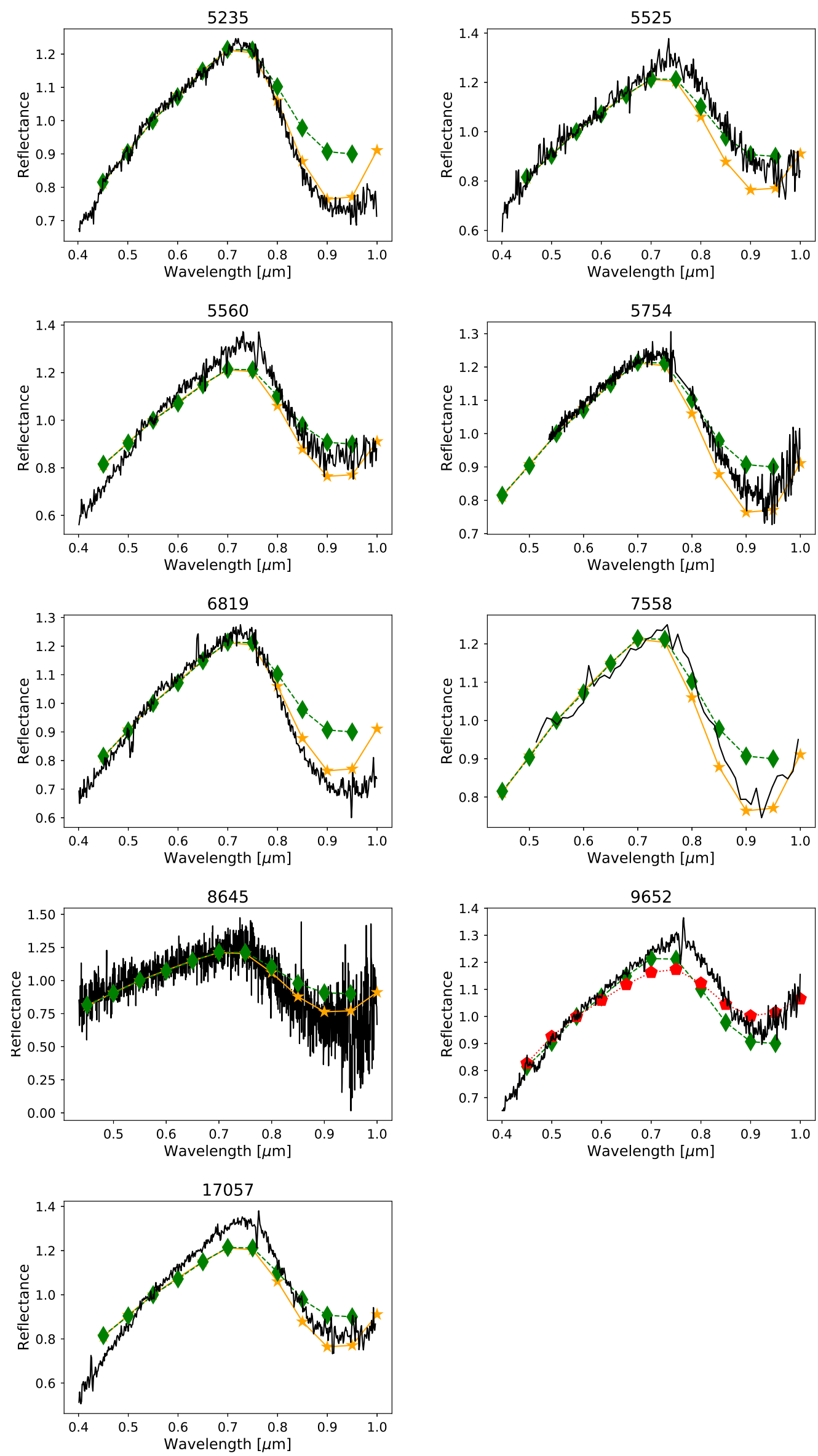

Fig. 1. continued. 

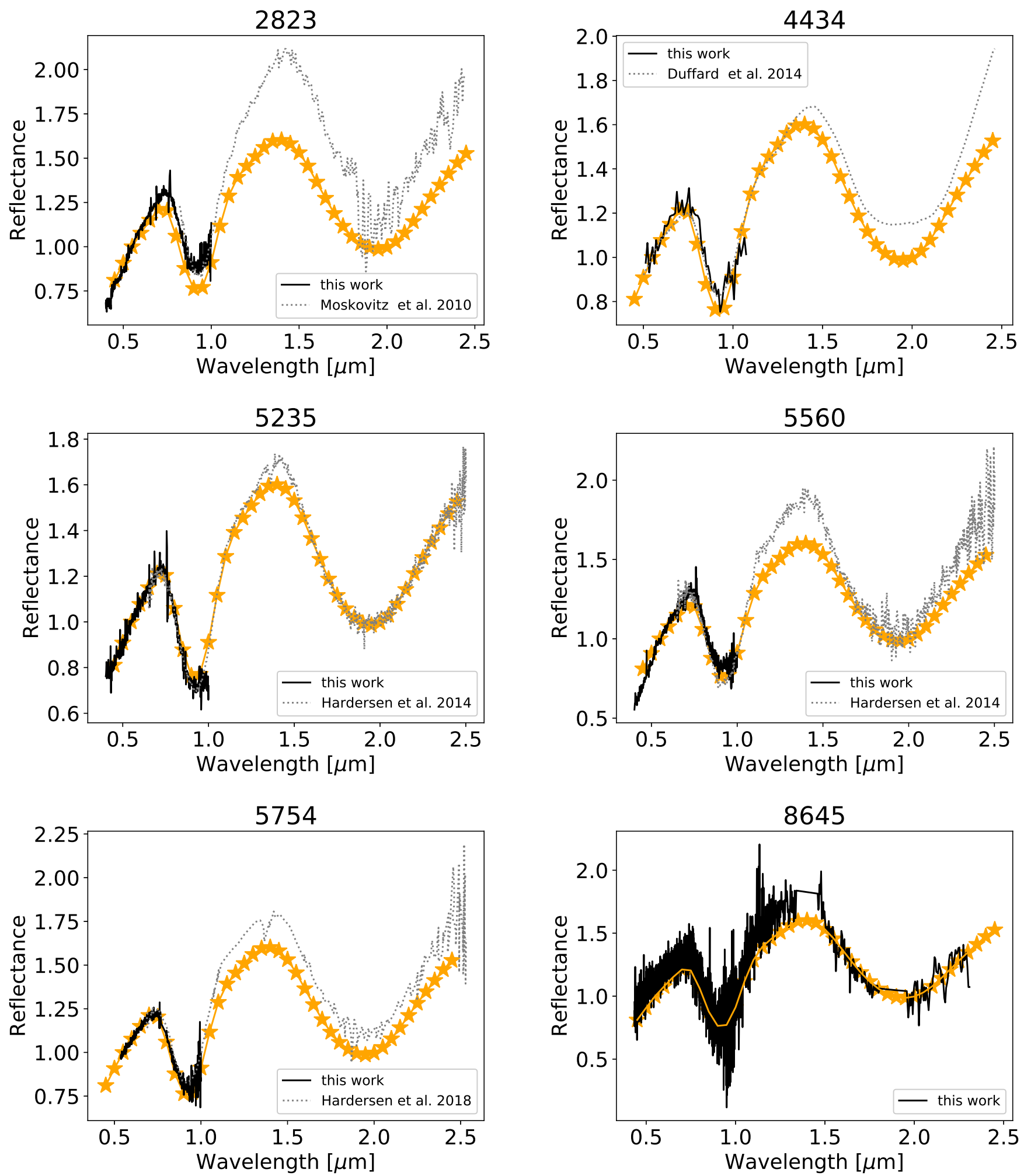

Fig. 2. Spectra obtained in this project for asteroids having both VIS and NIR spectra (NIR data obtained by us or from the literature). The orange line with stars represents the V-type template from DeMeo et al. (2009).

with diameters, geometric albedos, and the observed light curve amplitudes. For asteroids with unknown albedo we list the average value for the Vesta family of $p_{\mathrm{v}}=0.361 \pm 0.111$ (Masiero et al. 2013). A similar value is given by Thomas et al. (2011) for main belt V-types. Usui et al. (2012) gives lower average value of $p_{\mathrm{v}}=0.297 \pm 0.131$, but this estimate is based on only four objects.
Composite light curves for these objects are shown in Fig. A.1. The combined distribution of rotational properties of V-types in the inner main belt is derived in Sect. 4. All composite light curves were obtained using Fourier analysis, as in Kwiatkowski et al. (2010). For most of the observed asteroids the rotation periods were computed based on data spanning long time intervals, resulting in a synodic period averaged over 
Table 2. Taxonomic classification using the standard error method.

\begin{tabular}{|c|c|c|c|c|}
\hline Ast. num. & Tax. type & Dist. & Reliab. & Obs. night \\
\hline \multirow[t]{2}{*}{1914 Hartbeespoortdam } & V-type & 0.020 & $29.2 \%$ & 2019-04-22 \\
\hline & R-type & 0.046 & & \\
\hline \multirow[t]{2}{*}{2704 Julian Loewe } & V-type & 0.020 & $24.3 \%$ & 2018-04-03 \\
\hline & R-type & 0.058 & & \\
\hline \multirow[t]{2}{*}{2798 Vergilius } & R-type & 0.050 & $29.2 \%$ & $2018-04-22$ \\
\hline & V-type & 0.057 & & \\
\hline \multirow[t]{2}{*}{2823 Van der Laan } & V-type & 0.076 & $97.5 \%$ & 2019-06-09 \\
\hline & Sv-type & 0.161 & & \\
\hline \multirow[t]{2}{*}{3302 Schliemann } & Sv-type & 0.074 & $29.2 \%$ & 2019-06-09 \\
\hline & Sr-type & 0.079 & & \\
\hline \multirow[t]{2}{*}{3331 Kvitasberg } & V-type & 0.035 & $29.2 \%$ & 2019-04-22 \\
\hline & R-type & 0.060 & & \\
\hline \multirow[t]{2}{*}{4434 Nikulin } & V-type & 0.075 & $95.1 \%$ & 2018-04-03 \\
\hline & R-type & 0.120 & & \\
\hline \multirow[t]{2}{*}{5037 Habing } & V-type & 0.057 & $29.2 \%$ & $16-09-2016$ \\
\hline & R-type & 0.075 & & \\
\hline \multirow[t]{2}{*}{5235 Jean-Loup } & V-type & 0.036 & $100 \%$ & $16-09-2016$ \\
\hline & Sv-type & 0.157 & & \\
\hline \multirow[t]{2}{*}{5525 (1991 TS4) } & R-type & 0.041 & $26.8 \%$ & $16-09-2016$ \\
\hline & V-type & 0.056 & & \\
\hline \multirow[t]{2}{*}{5560 Amytis } & V-type & 0.076 & $100 \%$ & 2018-09-16 \\
\hline & R-type & 0.185 & & \\
\hline \multirow[t]{2}{*}{5754 (1992 FR2) } & V-type & 0.04 & $100 \%$ & $2018-04-22$ \\
\hline & Sv-type & 0.135 & & \\
\hline \multirow[t]{2}{*}{6819 McGarvey } & V-type & 0.030 & $26.8 \%$ & 2018-09-16 \\
\hline & R-type & 0.084 & & \\
\hline \multirow[t]{2}{*}{7558 Yurlov } & R-type & 0.048 & $24.3 \%$ & 2018-04-03 \\
\hline & V-type & 0.067 & & \\
\hline \multirow[t]{2}{*}{8645 (1988 TN) } & V-type & 0.068 & $92.6 \%$ & 2018-09-16 \\
\hline & Sv-type & 0.189 & & \\
\hline \multirow[t]{2}{*}{9652 (1996 AF2) } & R-type & 0.046 & $29.2 \%$ & 2020-01-08 \\
\hline & Sr-type & 0.047 & & \\
\hline \multirow[t]{2}{*}{17057 (1999 GS4) } & R-type & 0.099 & $29.2 \%$ & 2018-09-16 \\
\hline & V-type & 0.109 & & \\
\hline
\end{tabular}

Notes. Columns are asteroid number, taxonomic type distance from the template, and reliability of the classification. For each spectrum we list the two best matches. When possible we supplemented the spectra with the NIR counterpart from the literature.

Table 3. Spectral parameters: Slope A, Slope B, and apparent depth, as in Ieva et al. (2015), and without phase correction, center of the $1.0 \mu \mathrm{m}$ (BI) band.

\begin{tabular}{lllll}
\hline \hline Asteroid & $\begin{array}{l}\text { Slope A } \\
\left(\% / 10^{3} \AA\right)\end{array}$ & $\begin{array}{l}\text { Slope B } \\
\left(\% / 10^{3} \AA\right)\end{array}$ & $\begin{array}{l}\text { Depth } \\
-\end{array}$ & $\begin{array}{l}\text { BI } \\
(\mu \mathrm{m})\end{array}$ \\
\hline 1914 & 14.04 & -27.49 & 1.53 & 0.93 \\
2798 & 10.99 & -25.87 & 1.45 & 0.92 \\
2823 & 17.10 & -30.50 & 1.45 & 0.92 \\
3331 & 15.58 & -27.88 & 1.61 & 0.92 \\
5037 & 19.13 & -23.57 & 1.45 & 0.94 \\
5235 & 14.25 & -29.01 & 1.68 & 0.94 \\
5525 & 15.92 & -28.58 & 1.42 & 0.96 \\
5560 & 18.03 & -25.71 & 1.53 & 0.93 \\
5754 & 12.56 & -25.85 & 1.46 & 0.93 \\
6819 & 14.37 & -28.82 & 1.73 & 0.94 \\
9652 & 15.16 & -24.55 & 1.30 & 0.93 \\
17057 & 19.40 & -28.40 & 1.59 & 0.94 \\
\hline
\end{tabular}

that interval. Corresponding uncertainties are thus small and do not fully reflect the changes in synodic period. Therefore, we round the computed uncertainties to the first significant digit and provide the determined synodic periods to the same accuracy.

Asteroids (2247) Hiroshima, (2823) van der Laan, (5235) Jean-Loup, 7558 Yurlov, (8761) Crane, (9481) Menchu, and (9531) Jean-Luc are spectroscopically confirmed V-types (Wisniewski 1991; Moskovitz et al. 2008b, 2010; Sanctis et al. 2011; Solontoi et al. 2012; Hicks et al. 2014; Hardersen et al. 2014b, 2018). For (3331) Kvistaberg and (6819) McGarvey, we confirmed their V-type classification. Objects (7798) 1996 CL, (22113) 2000 RH9, and (27192) Senegali are putative V-types (Roig \& Gil-Hutton 2006; Carvano et al. 2010; Hasselmann et al. 2012; Licandro et al. 2017). Asteroid (5037) Habing was indicated by Carvano et al. (2010) and Hasselmann et al. (2012) as a $\mathrm{QV}_{\mathrm{p}}$ type. In our work and that of Solontoi et al. (2012), its spectrum shows a deep $0.9 \mu \mathrm{m}$ absorption band, typical of V-types.

\section{Statistical analysis of rotational periods of V-type and V-type candidate asteroids}

We supplement the rotational periods estimated in Sect. 3 with those known from the literature for spectroscopically confirmed V-types (V), V-type candidates $\left(\mathrm{V}_{\mathrm{c}}\right.$ ), and dynamical Vesta family members (assumed to be V-types) to perform statistical analysis. Since the V-type candidates have high confirmation rates, as shown in Sect. 3, we consider this to be a reasonable approach that does not skew our statistics substantially.

Our sample contains 536 asteroids (Tables A.2-A.7), including 428 Vesta family members (Nesvornỳ 2015), and 108 outside the Vesta family. In total, there are 155 spectroscopically confirmed V-types and $131 \mathrm{~V}$-type candidates. The remaining objects are dynamical Vesta family members assumed to be V-types (Moskovitz et al. 2008b estimate that $\sim 20 \%$ of the Vesta family are interlopers). We list them in Tables A.2-A.7 together with their synodic period, diameter, proper (or osculating for near-Earth asteroids, hereafter NEAs) orbital elements, and classification. Around $15 \%$ of NEAs are considered binary (Pravec et al. 1999; Pravec \& Harris 2007; Bottke \& Melosh 1996). A similar fraction is expected among small MBAs $(d<$ $10 \mathrm{~km})$. Since most V-types are smaller than $10 \mathrm{~km}$ in diameter, we expect around $15 \%$ binaries in our sample. However only 12 objects (3703, 3782, 5474, 5481, 18527 (Vesta family) 809, 2486, 5037, 32008 (Vesta fugitives) 854, 4383 (inner other group) 164121 (near-Earth)) are confirmed binaries. Thus, our sample may contain a larger number of undiscovered binaries.

Asteroids of known spectral type other than V-type were excluded from our analysis (e.g., those in the Vesta family were assumed to be interlopers). Spectral candidates are taken from Carvano et al. (2010), Hasselmann et al. (2012), Solontoi et al. (2012), Moskovitz et al. (2008b), and Licandro et al. (2017).

We subdivide our sample into several dynamically important groups, similarly to Ieva et al. (2018):

- Vesta family: Vesta family members, as defined by Nesvornỳ (2015) using the hierarchical clustering method (having orbital elements within ranges $2.26 \mathrm{au}<a<2.48 \mathrm{au}, 0.03<$ $e<0.16,5^{\circ}<i<8.3^{\circ}$ ). This sample contains 27 spectrally confirmed, 89 candidate, and 312 assumed V-types.

- Vesta fugitives: asteroids that escaped the Vesta family borders and are no longer identifiable as Vesta family members using the traditional clustering methods. Following the definition of Nesvorný et al. (2008), a Vesta family fugitive is a 
Table 4. Asteroid synodic periods and amplitudes found within this project.

\begin{tabular}{lcccc}
\hline \hline Asteroid no. and name & $\begin{array}{c}\text { Diameter } \\
(\mathrm{km})\end{array}$ & $\begin{array}{c}\text { Geometric } \\
\text { albedo }\end{array}$ & $\begin{array}{c}\text { Amplitude } \\
\text { (this work) } \\
(\mathrm{mag})\end{array}$ & $\begin{array}{c}\text { Period } \\
\text { (this work) } \\
(\mathrm{h})\end{array}$ \\
\hline (1914) Hartbeespoortdam & $9.56 \pm 0.19$ & $0.21 \pm 0.03$ & 0.04 & $6.3406 \pm 0.0002$ \\
(2247) Hiroshima & $4.37 \pm 0.10$ & $0.47 \pm 0.10$ & 0.14 & $4.4972 \pm 0.0002$ \\
(2442) Corbett & $8.33 \pm 0.38$ & $0.26 \pm 0.04$ & 0.19 & $11.456 \pm 0.001$ \\
(2486) Metsahovi & $7.88 \pm 0.06$ & $0.27 \pm 0.03$ & 0.13 & $4.4499 \pm 0.0002$ \\
(2823) van der Laan & $5.36 \pm 0.06$ & $0.32 \pm 0.05$ & 0.07 & $2.6262 \pm 0.0001$ \\
(3331) Kvistaberg & $4.62 \pm 0.93$ & $0.36 \pm 0.11$ & 0.16 & $7.5888 \pm 0.0001$ \\
(4188) Kitezh & $6.59 \pm 0.16$ & $0.34 \pm 0.04$ & 0.23 & $2.5820 \pm 0.0001$ \\
(5037) Habing ${ }^{(*)}$ & $5.68 \pm 0.08$ & $0.32 \pm 0.07$ & 0.29 & $2.8290 \pm 0.0001$ \\
(5235) Jean-Loup & $6.71 \pm 0.11$ & $0.36 \pm 0.06$ & 0.10 & $2.4524 \pm 0.0001$ \\
(6819) McGarvey & $4.68 \pm 0.22$ & $0.24 \pm 0.06$ & 0.31 & $2.7247 \pm 0.0001$ \\
(7558) Yurlov & $4.62 \pm 0.93$ & $0.36 \pm 0.11$ & 0.15 & $4.1157 \pm 0.0001$ \\
(7798) 1996 CL & $4.96 \pm 0.73$ & $0.29 \pm 0.11$ & 0.60 & $3.8724 \pm 0.0001$ \\
(8645) 1988 TN & $5.08 \pm 0.33$ & $0.30 \pm 0.07$ & 0.48 & $7.6250 \pm 0.0001$ \\
(8761) Crane & $3.84 \pm 0.78$ & $0.36 \pm 0.11$ & 0.23 & $2.6379 \pm 0.0001$ \\
(9481) Menchu & $4.67 \pm 0.04$ & $0.20 \pm 0.05$ & 0.15 & $3.0519 \pm 0.0001$ \\
(9531) Jean-Luc & $4.18 \pm 0.23$ & $0.28 \pm 0.05$ & 0.08 & $2.4999 \pm 0.0001$ \\
(22113) 2000 RH9 & $3.05 \pm 0.62$ & $0.36 \pm 0.11$ & 0.23 & $6.7425 \pm 0.0004$ \\
(27192) Senegali & $3.35 \pm 0.68$ & $0.36 \pm 0.11$ & 0.09 & $5.5741 \pm 0.0001$ \\
\hline
\end{tabular}

Notes. Albedos and diameters are from the astorb database (https://asteroid.lowell.edu/astinfo/). For asteroids with unknown albedo an average value of $p_{\mathrm{v}}=0.361 \pm 0.11$ (Masiero et al. 2013) was assumed and the diameter was computed based on the assumed albedo and absolute magnitude from JPL (https://ssd. jpl . nasa. gov/sbdb. cgi); these objects are highlighted in italics. ${ }^{(*)}$ We reported (5037) Habing as a binary in CBET 4730 based on 2020 opposition data.

V-type asteroid with $a<2.3$ au and a comparable $e$ and $i$ to the Vesta family. This sample contains 19 confirmed and 16 putative V-types.

- Low-i: Low inclination V-types with a comparable semimajor axis to the Vesta family and smaller inclinations $\left(i<6^{\circ}\right.$ and $2.3 \mathrm{au}<a<2.5 \mathrm{au}$, as defined by Nesvorný et al. 2008). There are 15 confirmed and 12 putative $\mathrm{V}$-types in this group.

- Inner Other (IO): the remaining V-type asteroids in the inner main belt were identified as IOs $(a<2.5 \mathrm{au})$. There are 10 confirmed and 12 putative V-types.

- V-type NEAs: a V-type asteroid in the near-Earth region (with a perihelion $q<1.3 \mathrm{au}$ ). This sample contains 20 confirmed and 1 putative V-type.

- Middle outer V-type (MOV): an asteroid located in the middle and outer main belt $(2.5 \mathrm{au}<a<5.0 \mathrm{au})$. There is only one spectrally confirmed MOV.

This division reflects interesting regions that may show various YORP signatures.

\subsection{Signatures of the YORP effect}

In Fig. A.1, we present the frequency distributions for the different subgroups. It should be noted that there are known observational biases working against small-amplitude and long-period asteroids (Marciniak et al. 2015, 2018) that are challenging to take into account. However, longer periods contribute to the low frequency bins amplifying the conclusions on YORP affecting the distribution. Small-amplitude objects may contribute to all bins. Rotations of the primaries of the binary systems were added into the frequency distributions. They mostly contribute to the fast-rotating bins and to the area near the spin barrier limit in Fig. 4, thus helping to constrain it.
The rotational frequency distribution for the Vesta family varies from that of the general inner main belt population (Lupishko et al. 2019). A significantly smaller excess of slow rotators and almost no fast rotators are observed for the Vesta family. The number of slowest rotating asteroids $\left(\omega \sim 0-1 \mathrm{~d}^{-1}\right)$ in the general inner main belt population (Lupishko et al. 2019) is about the same as in the peak bin $\left(\omega \sim 4-5 \mathrm{~d}^{-1}\right.$, which coincides with the rotation of (4) Vesta), whereas for the Vesta family we observe around $\sim 50 \%$ fewer asteroids among the slowest rotators than in the peak bin. Fast rotators from the last three bins constitute around $30 \%$ of the peak bin in the general population and around $20 \%$ of the peak bin in the Vesta family. However, we note that the size distribution varies between objects in this study $(0.7-10 \mathrm{~km})$ and that of Lupishko et al. (2019) $(3-15 \mathrm{~km})$, and therefore differences are expected.

Similarly the frequency distribution for V-types outside the Vesta family varies from that of the general inner main belt population (Lupishko et al. 2019). Slow rotators constitute around $50 \%$ of the peak bin and fast rotators around $50 \%$ of the peak bin. Therefore, there is a smaller excess of slow rotators among V-types outside the Vesta family than in the general inner main belt population and larger excess of fast rotators.

The population of V-types outside the Vesta family (and its sub-populations: fugitives and low- $i$ ) shows a surplus of slow and fast rotating objects compared to a Maxwellian distribution. The fraction of fast rotators is also larger than that in the Vesta family. However, the fugitives and low- $i$ constitute a small sample, thus valid conclusions can only be made for the collective population of V-types outside the Vesta family.

Interestingly, fewer fast rotators are observed in the Vesta family than in the population outside the family. This is likely due to the YORP effect spinning up small irregular asteroids, 

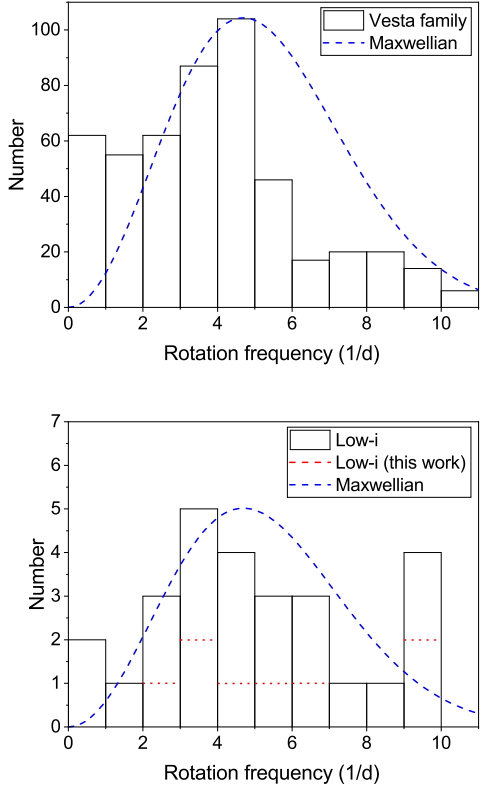
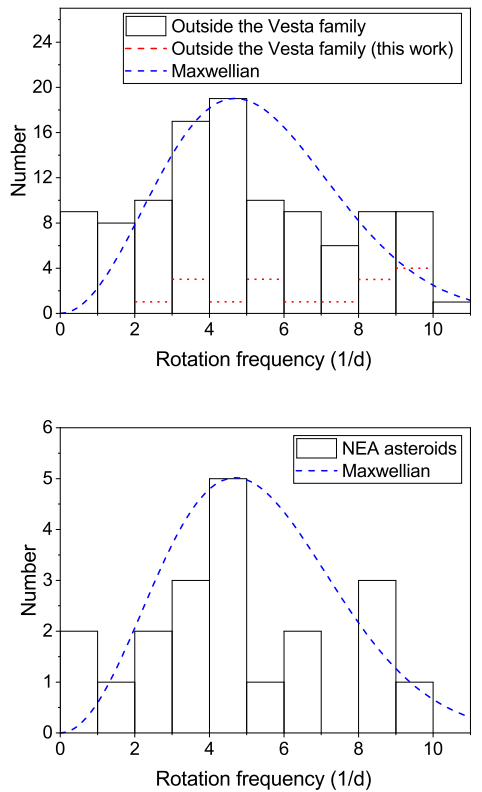
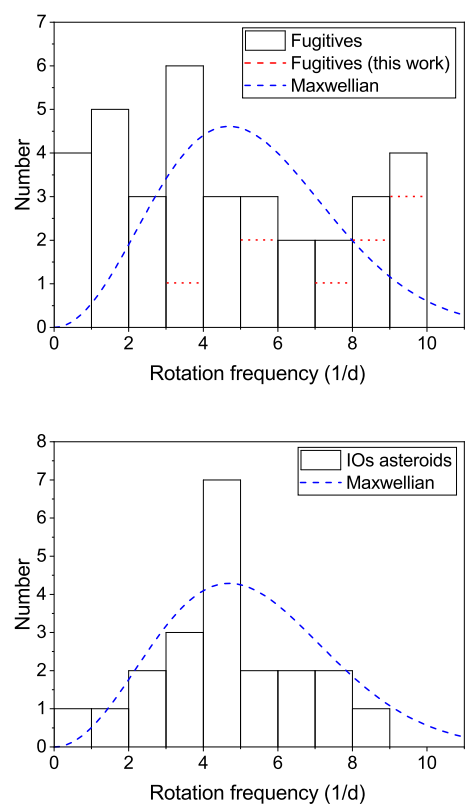

Fig. 3. Number vs. rotation frequency (in cycles per day) for different V-type populations. Rotation periods from LCDB (Warner et al. 2009b) and this work.

which are also more likely to migrate (via the Yarkovsky drift and resonances) outside the Vesta family, leaving more YORPmodification resistant objects (i.e., larger or more regularly shaped) in the core of the family. Farinella et al. (1998) showed that the Yarkovsky drift for basaltic objects is optimized for objects with diameters of $\sim 10 \mathrm{~km}$. The diameters of the V-types outside the Vesta family vary from less than $1 \mathrm{~km}$ to around $10 \mathrm{~km}$, which is reasonably close in the range to the optimal diameter. We show the histograms for NEAs and IO in Fig. 3. Due to the low number of known periods in these populations no compelling conclusions can be made.

To verify the influence of adding candidates to the samples, we plotted the distributions exclusively for spectrally confirmed V-types. Due to the small number of spectrally confirmed Vtypes in the Vesta family we cannot judge the influence of the candidates on the final sample. For asteroids outside the Vesta family we still have a substantial excess of fast rotators and a small excess of slow rotators, thus the conclusions remain unchanged.

\subsection{Spin barrier}

In Fig. 4, we present the location of the spectroscopically confirmed and candidate V-type asteroids in the size-frequency space. The spectroscopically confirmed V-types offset to the left of the plot (toward smaller sizes) are NEAs. The visible lack of spectroscopically confirmed objects with intermediate sizes is likely due their low apparent brightness, making them difficult targets for spectroscopy. Generally, the rotational properties of our sample resemble those of the general asteroid population. This means that most V-types and V-type candidates have rotation periods that are greater than about $2.2 \mathrm{~h}$, with the majority being between 2.4 and $21 \mathrm{~h}$.

We determined the spin barrier in frequency-light curve amplitude space. Light curve amplitudes for each asteroid (with quality code $U$-code $\geq 2$ ) were taken from the online service by Warner et al. (2009b) and this work. The frequency plot is shown
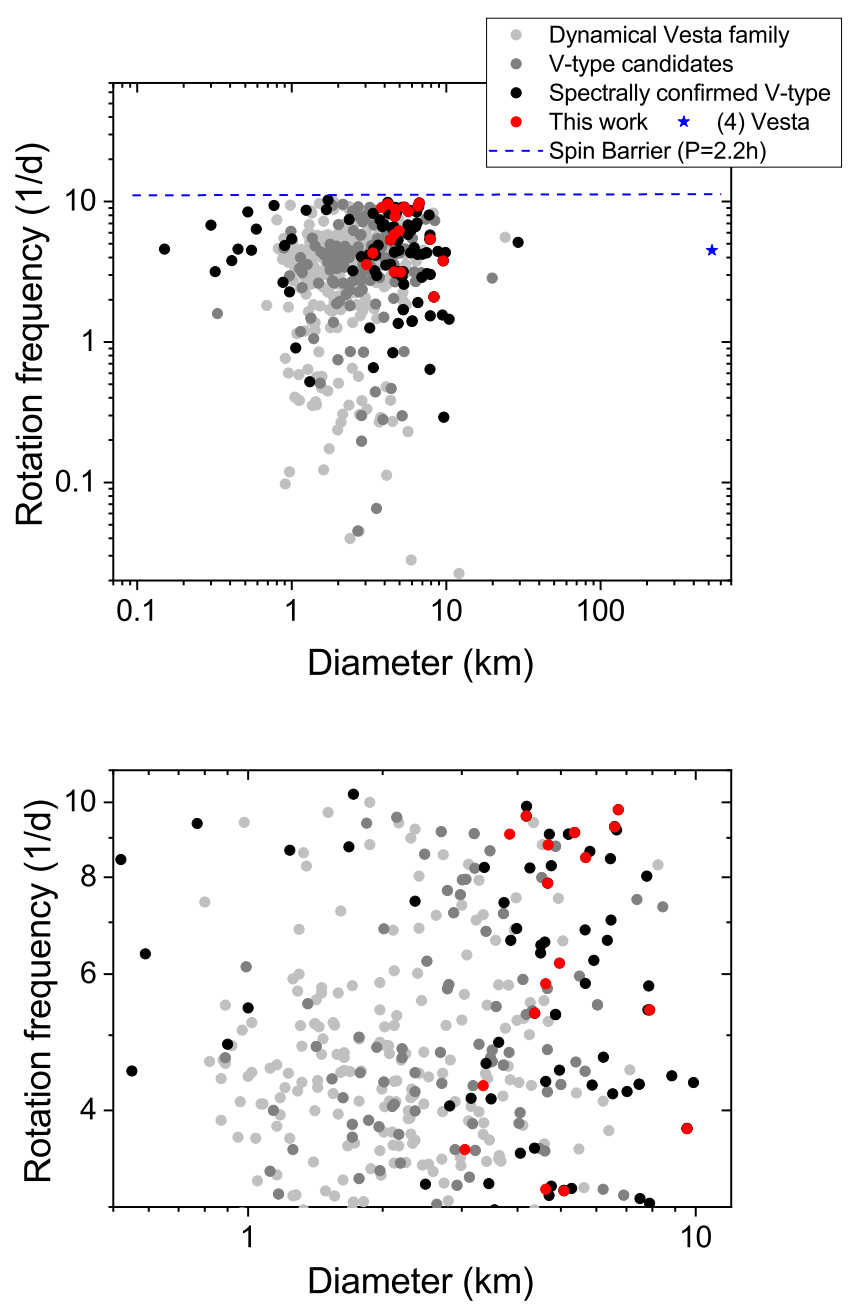

Fig. 4. Size-frequency distribution for dynamical Vesta family, V-types, and V-type candidates asteroids (top) and the same for the diameter range between 0.5 and $10 \mathrm{~km}$ (bottom). 


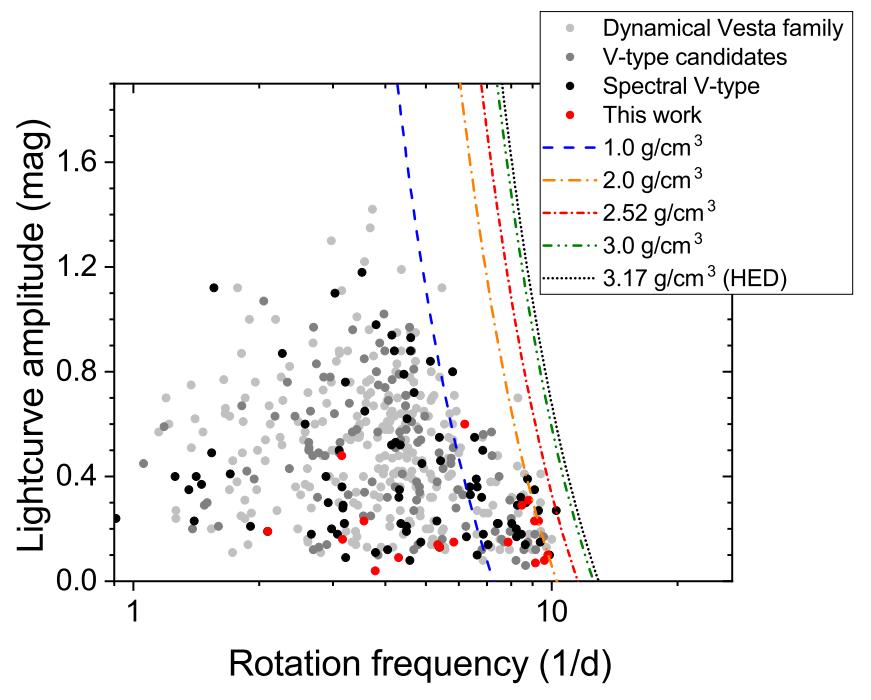

Fig. 5. Observed light curve amplitude vs. spin rate of V-type asteroids. The dashed curves are approximate upper limits of spin rates of bodies held together by self-gravitation only, with bulk densities plausible for asteroids.

in Fig. 5. The theoretical lines expected for various bulk densities according to Pravec \& Harris (2000) are indicated. The critical period corresponds to the period of a body with an ideal balance of the acceleration of gravity at the surface with the centrifugal acceleration at the equator (Pravec \& Harris 2000). The critical period depends on object elongation (and thus light curve amplitude) and density (both of which correlate with tensile strength). The derivation of the critical period can be found in Pravec \& Harris (2000).

The critical bulk density, estimated as $P_{\text {critical }}=\sqrt{3 \pi / G \rho} \approx$ $3.3 / \sqrt{\rho}$, exceeds the $2.0 \mathrm{~g} \mathrm{~cm}^{-3}$ estimated by Chang et al. (2015); one object is located at around $2.52 \mathrm{~g} \mathrm{~cm}^{-3}$. For comparison, the bulk density of the HED meteorites is around $3.17 \mathrm{~g} \mathrm{~cm}^{-3}$ (McSween et al. 2011).

\section{Summary}

We confirmed the taxonomic type of 17 objects (11 observed spectroscopically in the visible wavelengths for the first time) outside the Vesta family. We found that their spectral parameters are well within Vestoidal values.

We found synodic periods for 18 asteroids. We supplemented them with rotation periods from the literature and determined the distribution of rotational frequencies for V-types in the Vesta family and those outside the family. This sort of juxtaposition is presented for the first time for V-types in the literature.

The V-types outside the Vesta family show a significant surplus of fast rotators compared to the Vesta family. These might be objects evolved from the Vesta family that are more susceptible to both YORP and Yarkovsky effect. Thus, the YORP timescale must be comparable to the migration timescale for those objects.

We show for the first time that the spin barrier for the V-type asteroids exceeds the critical density of $2.0 \mathrm{~g} \mathrm{~cm}^{-3}$.

Acknowledgements. This work has been supported by grant No. 2017/26/D/ ST9/00240 from the National Science Centre, Poland. The work at Modra was supported by the Slovak Grant Agency for Science VEGA, Grant 1/0911/17. Based on observations made with $1.83 \mathrm{~m}$ Perkins and $1.07 \mathrm{~m}$ Hall and $0.79 \mathrm{~m}$ telescopes at Lowell Observatory, $2.24 \mathrm{~m}$ University of Hawaii telescope at Mauna Kea Observatory, $1.0 \mathrm{~m}$ Jacobus Kapteyn Telescope and 2.5 Nordic Optical Telescope at the Roque de los Muchachos Observatory on La Palma,
$4.3 \mathrm{~m}$ Lowell Discovery Telescope, $0.7 \mathrm{~m}$ Roman Baranowski telescope at Winer Observatory, $0.6 \mathrm{~m}$ Zeiss telescope at Modra Observatory, $0.8 \mathrm{~m}$ Odessa Multifunctional Telescope and $0.48 \mathrm{~m}$ AZT-3 telescope at Odessa-Mayaki Observatory. Some of the observations reported in this paper were obtained with the Southern African Large Telescope (SALT). Polish participation in SALT is funded by grant No. MNiSW DIR/WK/2016/07.

\section{References}

Alvarez-Candal, A., Duffard, R., Lazzaro, D., \& Michtchenko, T. 2006, A\&A, 459,969

Barucci, M. A., Capria, M. T., Coradini, A., \& Fulchignoni, M. 1987, Icarus, 72, 304

Binzel, R. P., \& Xu, S. 1993, Science, 260, 186

Binzel, R. P., Gehrels, T., \& M. S. Matthews, E. 1989, Asteroids II (Tucson: University of Arizona Press), 1139

Binzel, R. P., Harris, A. W., Bus, S. J., \& Burbine, T. H. 2001, Icarus, 151, 139

Binzel, R. P., Rivkin, A. S., Stuart, J. S., et al. 2004, Icarus, 170, 259

Birlan, M., Nedelcu, D. A., Descamps, P., et al. 2011, MNRAS, 415, 587

Birlan, M., Popescu, M., Irimiea, L., \& Binzel, R. 2016, AAS/Division for Planetary Sciences Meeting Abstracts, 48

Bottke, W. F., \& Melosh, H. J. 1996, Nature, 381, 51

Bottke, W. F., Nesvorný, D., Grimm, R. E., Morbidelli, A., \& O’brien, D. P. 2006a, Nature, 439, 821

Bottke, W. F., Vokrouhlickỳ, D., Rubincam, D. P., \& Nesvornỳ, D. 2006b, Ann. Rev. Earth Planet. Sci., 34, 157

Burbine, T. H., \& Binzel, R. P. 2002, Icarus, 159, 468

Burbine, T. H., Buchanan, P. C., Binzel, R. P., et al. 2001, Meteoritics Planet. Sci., 36, 761

Bus, S. J., \& Binzel, R. P. 2002, Icarus, 158, 106

Carruba, V., Michtchenko, T. A., Roig, F., Ferraz-Mello, S., \& Nesvornỳ, D. 2005, A\&A, 441, 819

Carruba, V., Michtchenko, T., \& Lazzaro, D. 2007, A\&A, 473, 967

Carruba, V., Huaman, M., Domingos, R., Santos, C. D., \& Souami, D. 2014, MNRAS, 439, 3168

Carry, B., Solano, E., Egg, S., \& DeMeo, F. E. 2016, Icarus, 268, 340

Carvano, J., Hasselmann, P., Lazzaro, D., \& Mothé-Diniz, T. 2010, A\&A, 510, A43

Chang, C.-K., Ip, W.-H., Lin, H.-W., et al. 2015, ApJS, 219, 27

Chang, C.-K., Lin, H.-W., Ip, W.-H., et al. 2019, AJ, 241, 15

Cloutis, E. A., Gaffey, M. J., Jackowski, T. L., \& Reed, K. L. 1986, J. Geophys. Res.: Solid Earth, 91, 11641

Cloutis, E., Izawa, M., Pompilio, L., et al. 2013, Icarus, 223, 850

Consolmagno, G. J., \& Drake, M. J. 1977, Geochim. Cosmochim. Acta, 41, 1271

Dandy, C. L., Fitzsimmons, A., \& Collander-Brown, S. J. 2003, Icarus, 163, 363

DeMeo, F., Binzel, R., Slivan, S., \& Bus, S. 2009, Icarus, 202, 160

De Sanctis, M. C., Combe, J.-P., Ammannito, E., et al. 2012, ApJ, 758, L36

De Sanctis, M. C., Ammannito, E., Capria, M. T., et al. 2013, Meteor. Planet. Sci., 48, 2166

Duffard, R., \& Roig, F. 2009, Planet. Space Sci., 57, 229

Duffard, R., Lazzaro, D., Licandro, J., et al. 2004, Icarus, 171, 120

Durech, J., Vokrouhlickỳ, D., Kaasalainen, M., et al. 2008, A\&A, 489, L25

Dymock, R., \& Miles, R. 2009, J. Br. Astron. Assoc., 119, 149

Erasmus, N., McNeill, A., Mommert, M., et al. 2018, ApJS, 237, 30

Erasmus, N., McNeill, A., Mommert, M., et al. 2019, AJ, 242, 12

Farinella, P., Vokrouhlickỳ, D., \& Hartmann, W. K. 1998, Icarus, 132, 378

Florczak, M., Lazzaro, D., \& Duffard, R. 2002, Icarus, 159, 178

Fulvio, D., Brunetto, R., Vernazza, P., \& Strazzulla, G. 2012, A\&A, 537, A11

Fulvio, D., Perna, D., Ieva, S., et al. 2016, MNRAS, 455, 584

Hammergren, M., Gyuk, G., \& Puckett, A. 2006, ArXiv preprint [arXiv:astro-ph/0609420]

Hardersen, P. S., Gaffey, M. J., \& Abell, P. A. 2004, Icarus, 167, 170

Hardersen, P. S., Reddy, V., Roberts, R., \& Mainzer, A. 2014, Icarus, 242, 269

Hardersen, P. S., Reddy, V., \& Roberts, R. 2015, ApJS, 221, 19

Hardersen, P. S., Reddy, V., Cloutis, E., et al. 2018, AJ, 156, 11

Harris, A. W. 1996, Lunar Planet. Sci. Conf., 27

Hasegawa, S., Miyasaka, S., Mito, H., et al. 2014, PASJ, 66

Hasegawa, S., Kuroda, D., Kitazato, K., et al. 2018, PASJ, 70

Hasselmann, P. H., Carvano, J. M., \& Lazzaro, D. 2012, SDSS-based Asteroid Taxonomy V1.1

Hicks, D., Buratti, B. J., Lawrence, K. J., et al. 2014, Icarus, 235, 60

Hiroi, T., Binzel, R. P., Sunshine, J. M., Pieters, C. M., \& Takeda, H. 1995, Icarus, 115,374

Howell, E. S., Merenyi, E., \& Lebofsky, L. A. 1994, J. Geophys. Res., 99, 10847

Huaman, M. E., Carruba, V., \& Domingos, R. C. 2014, MNRAS, 444, 2985

Ieva, S., Dotto, E., Lazzaro, D., et al. 2015, MNRAS, 455, 2871

Ieva, S., Dotto, E., Lazzaro, D., et al. 2018, MNRAS, 479, 2607 


\section{Oszkiewicz et al.: Spin rates of V-types}

Kaasalainen, M., \& Durech, J. 2007, in Proc. IAU Symp., 236

Kryszczyńska, A., Colas, F., Polińska, M., et al. 2012, A\&A, 546, A72

Kwiatkowski, T., Buckley, D., O'Donoghue, D., et al. 2010, A\&A, 509, A11

Lazzaro, D., Michtchenko, T., Carvano, J., et al. 2000, Science, 288, 2033

Lazzaro, D., Angeli, C., Carvano, J., et al. 2004, Icarus, 172, 179

Leith, T. B., Moskovitz, N. A., Mayne, R. G., et al. 2017, Icarus, 295, 61

Licandro, J., Popescu, M., Morate, D., \& de León, J. 2017, A\&A, 600, A126

Lin, C.-H., Ip, W.-H., Lin, Z.-Y., et al. 2018, Planet. Space Sci., 152, 116

Lowry, S. C., Fitzsimmons, A., Pravec, P., et al. 2007, Science, 316, 272

Lupishko, D., Mikhalchenko, O., \& Chiorny, V. 2019, Solar Syst. Res., 53, 208

Marciniak, A., Pilcher, F., Oszkiewicz, D., et al. 2015, Planet. Space Sci., 118, 256

Marciniak, A., Bartczak, P., Müller, T., et al. 2018, A\&A, 610, A7

Masiero, J. R., Mainzer, A., Bauer, J., et al. 2013, ApJ, 770, 7

McCord, T. B., Adams, J. B., \& Johnson, T. V. 1970, Science, 168, 1445

McSween, H. Y., Mittlefehldt, D. W., Beck, A. W., Mayne, R. G., \& McCoy, T. J. 2011, Space Sci. Rev., 163, 141

McSween, H. Y., Binzel, R. P., De Sanctis, M. C., et al. 2013, Meteor. Planet. Sci., 48, 2090

Medeiros, H., de León, J., Lazzaro, D., et al. 2019, MNRAS, 488, 3866

Michtchenko, T., Lazzaro, D., Ferraz-Mello, S., \& Roig, F. 2002, Icarus, 158, 343

Migliorini, A., De Sanctis, M., Lazzaro, D., \& Ammannito, E. 2017, MNRAS, 475, 353

Milani, A., Cellino, A., Knežević, Z., et al. 2014, Icarus, 239, 46

Moskovitz, N. A., Lawrence, S., Jedicke, R., et al. 2008a, ApJ, 682, L57

Moskovitz, N. A., Jedicke, R., Gaidos, E., et al. 2008b, Icarus, 198, 77

Moskovitz, N. A., Willman, M., Burbine, T. H., Binzel, R. P., \& Bus, S. J. 2010, Icarus, 208, 773

Neese, C., E. 2010, Asteroid Taxonomy V6.0

Nesvornỳ, D. 2015, Nesvorny HCM Asteroid Families V3.0, eAR-AVARGBDET-5-NESVORNYFAM-V3.0

Nesvorný, D., Roig, F., Gladman, B., et al. 2008, Icarus, 193, 85

Novakovic, B., Knezevic, Z., \& Milani, A. 2017, Synthetic Proper Elements, 524214 numbered-multiopp ast

Oszkiewicz, D. A., Kwiatkowski, T., Tomov, T., et al. 2014, A\&A, 572, A29

Oszkiewicz, D., Kankiewicz, P., Włodarczyk, I., \& Kryszczyńska, A. 2015, A\&A, 584, A18

Oszkiewicz, D. A., Skiff, B. A., Moskovitz, N., et al. 2017, A\&A, 599, A107

Oszkiewicz, D., Kryszczynska, A., Kankiewicz, P., et al. 2019, A\&A, 623, A170

Pajuelo, M., Birlan, M., Carry, B., et al. 2018, MNRAS, 477, 5590
Popescu, M., Birlan, M., Binzel, R., et al. 2011, A\&A, 535, A15

Popescu, M., Birlan, M., \& Nedelcu, D. A. 2012, A\&A, 544, A130

Popescu, M., Licandro, J., Carvano, J. M., et al. 2018, A\&A, 617, A12

Pravec, P., \& Harris, A. W. 2000, Icarus, 148, 12

Pravec, P., \& Harris, A. 2007, Icarus, 190, 250

Pravec, P., Wolf, M., \& Sarounová, L. 1999, in Evolution and Source Regions of Asteroids and Comets, IAU Colloq., 173, 159

Pravec, P., Harris, A., \& Michalowski, T. 2002, Asteroids III (Berlin: Springer), 113

Pravec, P., Harris, A. W., Vokrouhlický, D., et al. 2008, Icarus, 197, 497

Roatsch, T., Kersten, E., Matz, K.-D., et al. 2012, Planet. Space Sci., 73, 283

Roig, F., \& Gil-Hutton, R. 2006, Icarus, 183, 411

Roig, F., Nesvornỳ, D., Gil-Hutton, R., \& Lazzaro, D. 2008, Icarus, 194, 125

Rubincam, D. P. 2000, Icarus, 148, 2

Russell, C., Raymond, C., Coradini, A., et al. 2012, Science, 336, 684

Salo, H. 1987, Icarus, 70, 37

Sanchez, J. A., Michelsen, R., Reddy, V., \& Nathues, A. 2013, Icarus, 225, 131

Sanctis, M. C. D., Migliorini, A., Jasmin, F. L., et al. 2011, A\&A, 533, A77

Schenk, P., O’Brien, D. P., Marchi, S., et al. 2012, Science, 336, 694

Scott, E. R., Greenwood, R. C., Franchi, I. A., \& Sanders, I. S. 2009, Geochim. Cosmochim. Acta, 73, 5835

Slivan, S. M., Binzel, R. P., da Silva, L. D. C., et al. 2003, Icarus, 162, 285

Slivan, S. M., Binzel, R. P., Kaasalainen, M., et al. 2009, Icarus, 200, 514

Solontoi, M. R., Hammergren, M., Gyuk, G., \& Puckett, A. 2012, Icarus, 220, 577

Spahr, T. B., Hergenrother, C. W., Larson, S. M., et al. 1997, Icarus, 129, 415

Spoto, F., Milani, A., \& Knežević, Z. 2015, Icarus, 257, 275

Sunshine, J. M., Pieters, C. M., \& Pratt, S. F. 1990, J. Geophys. Res.: Solid Earth, 95,6955

Taylor, P. A., Margot, J.-L., Vokrouhlickỳ, D., et al. 2007, Science, 316, 274

Thomas, P. C., Binzel, R. P., Gaffey, M. J., et al. 1997, Icarus, 128, 88

Thomas, C. A., Trilling, D. E., Emery, J., et al. 2011, AJ, 142, 85

Thomas, C. A., Emery, J. P., Trilling, D. E., et al. 2014, Icarus, 228, 217

Usui, F., Kasuga, T., Hasegawa, S., et al. 2012, AJ, 762, 56

Walsh, K. J., Richardson, D. C., \& Michel, P. 2008, Nature, 454, 188

Warner, B. D., Harris, A. W., Vokrouhlickỳ, D., Nesvornỳ, D., \& Bottke, W. F. 2009a, Icarus, 204, 172

Warner, B., Harris, A., \& Pravec, P. 2009b, Icarus, 202, 134

Wasson, J. T. 2013, Earth Planet. Sci. Lett., 381, 138

Wisniewski, W. Z. 1991, Icarus, 90, 117

Xu, S., Binzel, R. P., Burbine, T. H., \& Bus, S. J. 1995, Icarus, 115, 1 


\section{Appendix A: Additional tables and figures}

Table A.1. Observing setup and circumstances for VIS spectra.

\begin{tabular}{|c|c|c|c|c|c|c|c|c|c|c|}
\hline Asteroid & Telescope & Grism & $\begin{array}{l}\text { Slit width } \\
(\operatorname{arcsec})\end{array}$ & $\begin{array}{l}\text { Observing } \\
\text { night }\end{array}$ & $\begin{array}{l}\text { Phase } \\
\text { angle (deg) }\end{array}$ & $\begin{array}{l}\text { Air } \\
\text { mass }\end{array}$ & Solar Analog & $\begin{array}{l}\text { Airm } \\
\text { mass }\end{array}$ & $\begin{array}{l}\text { Ref. } \\
\text { VIS }\end{array}$ & $\begin{array}{l}\text { Ref. } \\
\text { NIR }\end{array}$ \\
\hline 1914 Hartbeespoortdam & NOT & 11 & 1.3 & 2019-04-22 & 14.3 & 1.32 & SA $110-361$ & 1.34 & (1) & (2) \\
\hline 2704 Julian Loewe & NOT & 11 & 1.3 & 2019-06-09 & 6.7 & 1.60 & HD 133011 & 1.47 & (3) & \\
\hline 2798 Vergilius & NOT & 11 & 1.3 & 2019-04-22 & 5.0 & 1.67 & SA102-1081 & 1.66 & & \\
\hline 2823 Van der Laan & NOT & 11 & 1.3 & 2019-06-09 & 6.2 & 1.59 & HD 140990 & 1.60 & & (4) \\
\hline 3302 Schliemann & NOT & 11 & 1.3 & 2019-06-09 & 26.1 & 1.49 & HD 203311 & 1.50 & & \\
\hline 3331 Kvitasberg & NOT & 11 & 1.3 & 2019-04-22 & 17.3 & 1.06 & HD 77730 & 1.08 & & \\
\hline 4434 Nikulin & NOT & 12 & 1.3 & 2018-04-03 & 9.0 & 1.26 & Land 107-998 & 1.24 & (3) & (5) \\
\hline 5037 Habing & NOT & 11 & 1.3 & 2018-09-16 & 14.7 & 1.41 & HD 210078 & 1.36 & (6) & \\
\hline 5235 Jean-Loup & NOT & 11 & 1.3 & $2018-09-16$ & 19.2 & 1.06 & $51 \mathrm{Peg}$ & 1.13 & & (7) \\
\hline 5525 (1991 TS4) & NOT & 11 & 1.3 & 2018-09-16 & 25.4 & 1.35 & Hyades 64 & 1.21 & (6) & \\
\hline 5560 Amytis & NOT & 11 & 1.3 & 2018-09-16 & 7.4 & 1.51 & HIP 11915 & 1.41 & (6) & (7) \\
\hline 5754 (1992 FR2) & SALT & PG0300 & 3.0 & 2017-11-27 & 17.5 & 1.22 & HD 11532 & 1.2 & & (8) \\
\hline 6819 McGarvey & NOT & 11 & 1.3 & 2018-09-16 & 17.9 & 1.58 & $51 \mathrm{Peg}$ & 1.34 & & \\
\hline 7558 Yurlov & NOT & 12 & 1.3 & 2018-04-03 & 27.2 & 1.32 & Land 102-1081 & 1.18 & (9) & \\
\hline \multirow[t]{2}{*}{8645 (1988 TN) } & LDT & $1501 / \mathrm{mm}$ & 3 & 2020-01-08 & 7.1 & 1.01 & SA 102-1081 & 1.43 & & \\
\hline & LDT & PRISM & 1.34 & 2020-01-08 & 7.1 & 1.05 & SA $102-1081$ & 1.4 & & \\
\hline 9652 (1996 AF2) & NOT & 11 & 1.3 & 2019-04-22 & 9.3 & 1.43 & HD 117860 & 1.44 & & \\
\hline 17057 (1999 GS4) & NOT & 11 & 1.3 & 2018-09-16 & 15.5 & 1.32 & HD 209847 & 1.36 & & \\
\hline
\end{tabular}

Notes. Columns are: asteroid designation, telescope, grisms, slit width, observing night, asteroid phase angle, asteroid air mass at the start of exposures, solar analogs used, air masses of the solar analogs at the start of exposures. References are given for the first publication of each asteroid spectrum.

References. (1) Alvarez-Candal et al. (2006), (2) Fulvio et al. (2016), (3) Bus \& Binzel (2002), (4) Moskovitz et al. (2010), (5) Duffard et al. (2004), (6) Solontoi et al. (2012), (7) Hardersen et al. (2014b), (8) Hardersen et al. (2018), (9) Moskovitz et al. (2008b).

Table A.2. Spectral V-types and V-type candidates $\left(\mathrm{V}_{\mathrm{c}}\right)$ in the Vesta family population, their physical and orbital parameters.

\begin{tabular}{|c|c|c|c|c|c|c|c|}
\hline $\begin{array}{r}\text { Asteroid } \\
\text { No. }\end{array}$ & $\begin{array}{l}\text { Synodic period } \\
\text { (LCDB) }(\mathrm{h})\end{array}$ & $\begin{array}{l}\text { U-code } \\
\text { (LCDB) }\end{array}$ & $\begin{array}{r}\text { Diameter } \\
(\mathrm{km})\end{array}$ & $\begin{array}{l}\text { Class, } \\
\text { references }\end{array}$ & $\begin{array}{c}a \\
(\mathrm{au})\end{array}$ & $e$ & $\sin (i)$ \\
\hline (4) Vesta & 5.34212766 & 3 & 525.4 & $\left.\mathrm{~V}^{(1,14,15,16,17,18,19}\right)$ & 2.362 & 0.099 & 0.1113 \\
\hline 1929 & 2.9887 & 3 & 7.8 & $\mathrm{~V}^{(1,10,17,18,19)}$ & 2.363 & 0.114 & 0.1232 \\
\hline 1933 & 3.671 & 3 & 4.5 & $\mathrm{~V}^{(1,9,10)}$ & 2.353 & 0.094 & 0.1188 \\
\hline 1959 & 7.726 & 3 & 6.9 & $\mathrm{~V}_{\mathrm{c}}^{(12,21)}$ & 2.316 & 0.095 & 0.1193 \\
\hline 1979 & 7.5209 & 3 & 4.8 & $\mathrm{~V}^{(5,12,21)}, \mathrm{QV}^{(2,13)}$ & 2.374 & 0.102 & 0.1136 \\
\hline 2011 & 8.209 & 3 & 5.2 & $\mathbf{V}^{(2,6,8,9,12,13,21)}, \mathbf{J}^{(1,10)}$ & 2.387 & 0.111 & 0.1110 \\
\hline 2045 & 82.4 & 2 & 9.7 & $\mathrm{~V}^{(1,17,18,19)}$ & 2.380 & 0.090 & 0.1164 \\
\hline 2468 & 5.122 & 3 & 6.2 & $\mathrm{~V}^{(1,17)}$ & 2.327 & 0.115 & 0.1122 \\
\hline 2508 & 17.7 & $2-$ & 4.9 & $\mathrm{~V}^{(1,9,11,12,17,21)}$ & 2.368 & 0.094 & 0.1124 \\
\hline 2511 & 4.144 & 3 & 7.9 & $\mathrm{~V}^{(1,17,19)}$ & 2.299 & 0.095 & 0.1271 \\
\hline 2590 & 15.59 & 3 & 7.9 & $\mathrm{~V}^{(1,3,10)}$ & 2.343 & 0.097 & 0.1177 \\
\hline 2974 & 856.0 & $2+$ & 5.9 & - & 2.312 & 0.094 & 0.1236 \\
\hline 3155 & 8.31 & 3 & 7.0 & $\mathrm{~V}^{(1,17,18,19)}, \mathrm{J}^{(10,22)}$ & 2.343 & 0.098 & 0.1165 \\
\hline 3263 & 3.193 & $2+$ & 4.9 & - & 2.414 & 0.085 & 0.1198 \\
\hline 3268 & 17.0 & 2 & 6.0 & $\mathrm{~V}^{(1,20)}$ & 2.347 & 0.100 & 0.1223 \\
\hline 3281 & 6.7295 & 3 & 5.5 & - & 2.350 & 0.122 & 0.1074 \\
\hline 3376 & 7.916 & $3-$ & 7.9 & $\mathrm{~V}^{(2,11,13)}, \mathrm{Sq}^{(1,17)}$ & 2.349 & 0.096 & 0.1232 \\
\hline 3703 & 3.235 & 3 & 3.7 & $\mathrm{~V}^{(3,19)}$ & 2.332 & 0.091 & 0.1183 \\
\hline
\end{tabular}

Notes. Proper elements are from Novakovic et al. (2017): $a$ - proper semi-major axis, $e$ - proper eccentricity, $\sin (i)$ - sine of proper inclination. References. Taxonomic classification/candidacy is from: ${ }^{(1)}$ Neese (2010), ${ }^{(2)}$ Hasselmann et al. (2012), ${ }^{(3)}$ Wisniewski (1991), ${ }^{(4)}$ Solontoi et al. (2012), ${ }^{(5)}$ Oszkiewicz et al. (2014), ${ }^{(6)}$ Sanctis et al. (2011), ${ }^{(7)}$ Hardersen et al. $(2018),{ }^{(8)}$ Hardersen et al. $(2015)$, ${ }^{(9)}$ Hasegawa et al. (2014), ${ }^{(10)}$ Xu et al. (1995), ${ }^{(11)}$ Spahr et al. (1997), ${ }^{(12)}$ Licandro et al. (2017), ${ }^{(13)}$ Carvano et al. (2010), ${ }^{(14)}$ Binzel et al. (1989), ${ }^{(15)}$ Barucci et al. (1987), ${ }^{(16)} \mathrm{Howell} \mathrm{et} \mathrm{al.} \mathrm{(1994),}$ ${ }^{(17)}$ Bus \& Binzel (2002), ${ }^{(18)}$ DeMeo et al. (2009), ${ }^{(19)}$ Moskovitz et al. (2010), ${ }^{(20)}$ Hicks et al. (2014), ${ }^{(21)}$ Popescu et al. (2018), ${ }^{(22)}$ Binzel \& Xu (1993),

${ }^{(23)}$ Lazzaro et al. (2004), ${ }^{(24)}$ Chang et al. (2019), ${ }^{(25)}$ Erasmus et al. (2018), ${ }^{(26)}$ Erasmus et al. (2019). 
D. Oszkiewicz et al.: Spin rates of V-types

Table A.2. continued.

\begin{tabular}{|c|c|c|c|c|c|c|c|}
\hline $\begin{array}{r}\text { Asteroid } \\
\text { No. }\end{array}$ & $\begin{array}{l}\text { Synodic period } \\
\text { (LCDB) }(\mathrm{h})\end{array}$ & $\begin{array}{l}\text { U-code } \\
(\mathrm{LCDB})\end{array}$ & $\begin{array}{r}\text { Diameter } \\
(\mathrm{km}) \\
\end{array}$ & $\begin{array}{l}\text { Class, } \\
\text { references }\end{array}$ & $\begin{array}{c}a \\
(\mathrm{au}) \\
\end{array}$ & $e$ & $\sin (i)$ \\
\hline 3720 & 4.79 & $2+$ & 4.3 & - & 2.320 & 0.093 & 0.1139 \\
\hline 3782 & 3.84 & 3 & 5.9 & $\mathrm{~V}^{(1,2,7,13,17,19)}$ & 2.416 & 0.108 & 0.1063 \\
\hline 3936 & 6.6322 & 3 & 4.6 & - & 2.428 & 0.123 & 0.1112 \\
\hline 3944 & 5.565 & 2 & 5.9 & $\mathrm{~V}^{(1,10)}$ & 2.369 & 0.109 & 0.1177 \\
\hline 4215 & 12.6 & 2 & 6.6 & $\mathbf{V}^{(1,17,19)}, \mathbf{J}^{(10,22)}$ & 2.418 & 0.098 & 0.1152 \\
\hline 4524 & 1069.0 & 2 & 12.1 & - & 2.321 & 0.087 & 0.1291 \\
\hline 4228 & 2.772 & 2 & 3.5 & $\mathrm{~V}_{\mathrm{c}}^{(12,21)}$ & 2.304 & 0.090 & 0.1047 \\
\hline 4684 & 12.169 & $3-$ & 5.3 & - & 2.398 & 0.107 & 0.1025 \\
\hline 4852 & 9.715 & 2 & 4.0 & - & 2.304 & 0.095 & 0.1087 \\
\hline 5111 & 2.839 & 3 & 6.5 & $\mathrm{~V}^{(1,2,13,18,19)}, \mathrm{J}^{(17)}$ & 2.354 & 0.083 & 0.1168 \\
\hline 5240 & 5.673 & 3 & 7.0 & $\mathrm{~V}^{(1,17)}$ & 2.383 & 0.105 & 0.1108 \\
\hline 5307 & 5.343 & 3 & 5.7 & $\mathrm{~V}_{\mathrm{c}}^{(12,21)}$ & 2.415 & 0.122 & 0.1081 \\
\hline 5474 & 3.6242 & 3 & 5.1 & - & 2.383 & 0.105 & 0.1214 \\
\hline 5481 & 3.6196 & 3 & 3.9 & $\mathbf{V}^{(1,2,13,17,19,23)}$ & 2.340 & 0.093 & 0.1101 \\
\hline 5858 & 7.553 & 3 & 5.6 & - & 2.315 & 0.090 & 0.1135 \\
\hline 6003 & 104.406 & 2 & 5.7 & - & 2.344 & 0.094 & 0.1116 \\
\hline 6085 & 5.556 & 3 & 5.0 & $\mathrm{~V}_{\mathrm{c}}^{(12,21)}$ & 2.307 & 0.092 & 0.1243 \\
\hline 6131 & 6.77 & 2 & 4.6 & $\mathrm{~V}_{\mathrm{c}}^{(2,13)}$ & 2.423 & 0.125 & 0.1031 \\
\hline 6159 & 10.639 & 3 & 5.3 & - & 2.291 & 0.089 & 0.1105 \\
\hline 6331 & 9.333 & $2+$ & 5.3 & $\mathrm{~V}^{(2,9,13)}$ & 2.358 & 0.096 & 0.1230 \\
\hline 6418 & 6.377 & 3 & 6.4 & - & 2.260 & 0.108 & 0.1171 \\
\hline 6659 & 12.985 & 2 & 5.5 & - & 2.372 & 0.106 & 0.1045 \\
\hline 6877 & 4.1745 & 3 & 4.7 & $\mathrm{~V}_{\mathrm{c}}^{(2,12,13,21)}$ & 2.323 & 0.088 & 0.1224 \\
\hline 6944 & 2.9128 & 3 & 3.4 & $\mathrm{~V}^{(2,4,13)}$ & 2.316 & 0.108 & 0.1210 \\
\hline 6956 & 4.463 & 2 & 4.3 & $\mathrm{~V}_{\mathrm{c}}^{(2,13)}$ & 2.443 & 0.111 & 0.1100 \\
\hline 7012 & 8.938 & 2 & 4.7 & $\mathrm{~V}_{\mathrm{c}}^{(12)}$ & 2.312 & 0.095 & 0.1189 \\
\hline 7334 & 4.0659 & 3 & 4.1 & $\mathrm{~V}_{\mathrm{c}}^{(2,13)}$ & 2.413 & 0.109 & 0.1070 \\
\hline 7510 & 5.38 & 2 & 3.4 & - & 2.267 & 0.110 & 0.1129 \\
\hline 7767 & 85.658 & 2 & 3.9 & - & 2.372 & 0.099 & 0.1051 \\
\hline 7933 & 3.26 & $2+$ & 3.0 & - & 2.328 & 0.091 & 0.1188 \\
\hline 7946 & 51.343 & 2 & 4.4 & $\mathrm{~V}_{\mathrm{c}}^{(2,13)}$ & 2.370 & 0.097 & 0.1115 \\
\hline 8108 & 28.5696 & 2 & 4.5 & $\mathrm{~V}^{(2,4,13)}$ & 2.369 & 0.089 & 0.1252 \\
\hline 8359 & 2.89111 & 3 & 8.2 & - & 2.350 & 0.107 & 0.1206 \\
\hline 8692 & 4.698 & 2 & 3.8 & - & 2.354 & 0.094 & 0.1045 \\
\hline 9136 & 5.75 & 2 & 3.7 & - & 2.436 & 0.116 & 0.1105 \\
\hline 9199 & 5.322 & $2+$ & 7.1 & - & 2.401 & 0.094 & 0.1246 \\
\hline 9204 & 80.483 & 2 & 5.2 & $\mathrm{~V}_{\mathrm{c}}^{(2,12,13,21)}$ & 2.332 & 0.099 & 0.1195 \\
\hline 9220 & 6.04 & 2 & 4.1 & $\mathrm{~V}_{\mathrm{c}}^{(21)}$ & 2.406 & 0.102 & 0.1078 \\
\hline 9295 & 5.13 & $2-$ & 3.5 & $\mathrm{~V}_{\mathrm{c}}^{(2,13)}$ & 2.390 & 0.106 & 0.1089 \\
\hline 9325 & 4.607 & 2 & 4.7 & - & 2.429 & 0.102 & 0.1127 \\
\hline 9392 & 5.524 & 2 & 4.0 & - & 2.444 & 0.097 & 0.1064 \\
\hline 9616 & 5.018 & 2 & 3.5 & $\mathrm{~V}_{\mathrm{c}}^{(12,21)}$ & 2.422 & 0.109 & 0.1091 \\
\hline 9753 & 4.356 & 2 & 4.3 & - & 2.429 & 0.117 & 0.1019 \\
\hline 9872 & 11.598 & 2 & 3.0 & - & 2.369 & 0.109 & 0.1130 \\
\hline 10001 & 213.368 & 2 & 4.1 & - & 2.377 & 0.115 & 0.1166 \\
\hline 10022 & 4.564 & 2 & 4.1 & - & 2.369 & 0.111 & 0.1072 \\
\hline 10320 & 5.92 & 2 & 2.8 & $\mathrm{~V}^{(2,9,13)}$ & 2.288 & 0.089 & 0.1154 \\
\hline 10484 & 5.508 & 3 & 4.6 & $\mathrm{~V}^{(2,5,13)}$ & 2.321 & 0.100 & 0.1122 \\
\hline 10868 & 80.145 & 2 & 2.8 & $\mathrm{~V}_{\mathrm{c}}^{(2,13)}$ & 2.313 & 0.093 & 0.1124 \\
\hline 11697 & 2.805 & $2+$ & 2.7 & - & 2.343 & 0.097 & 0.1219 \\
\hline 11917 & 89.317 & 2 & 3.7 & - & 2.340 & 0.091 & 0.1190 \\
\hline 12088 & 3.342 & 2 & 3.7 & $\mathrm{~V}_{\mathrm{c}}^{(2,13)}$ & 2.355 & 0.106 & 0.1209 \\
\hline 12405 & 13.218 & 2 & 2.4 & $\mathrm{~V}_{\mathrm{c}}^{(2,13)}$ & 2.373 & 0.085 & 0.1046 \\
\hline 12578 & 6.008 & 2 & 2.3 & - & 2.301 & 0.091 & 0.1301 \\
\hline 12584 & 7.071 & 2 & 3.0 & - & 2.372 & 0.094 & 0.1180 \\
\hline
\end{tabular}


Table A.2. continued.

\begin{tabular}{|c|c|c|c|c|c|c|c|}
\hline $\begin{array}{r}\text { Asteroid } \\
\text { No. }\end{array}$ & $\begin{array}{r}\text { Synodic period } \\
.(\mathrm{LCDB})[\mathrm{h}]\end{array}$ & $\begin{array}{l}\text { U-code } \\
(\mathrm{LCDB})\end{array}$ & $\begin{array}{r}\text { Diameter } \\
{[\mathrm{km}]} \\
\end{array}$ & $\begin{array}{l}\text { Class, } \\
\text { references }\end{array}$ & $\begin{array}{c}a \\
{[\mathrm{au}]}\end{array}$ & $e$ & $\sin (i)$ \\
\hline 12619 & 5.847 & 2 & 2.9 & - & 2.455 & 0.109 & 0.1090 \\
\hline 12982 & 601.869 & 2 & 2.4 & - & 2.388 & 0.121 & 0.1018 \\
\hline 13058 & 4.299 & 2 & 24.1 & - & 2.358 & 0.109 & 0.1133 \\
\hline 13164 & 3.334 & 2 & 2.9 & $\mathrm{~V}_{\mathrm{c}}^{(12,21)}$ & 2.300 & 0.095 & 0.1215 \\
\hline 13243 & 88.4 & 2 & 4.5 & - & 2.288 & 0.098 & 0.1209 \\
\hline 13258 & 4.59 & 2 & 4.0 & - & 2.372 & 0.097 & 0.1165 \\
\hline 13262 & 8.35 & 2 & 3.7 & $\mathrm{~V}_{\mathrm{c}}^{(2,13)}$ & 2.377 & 0.100 & 0.0991 \\
\hline 13607 & 4.133 & 2 & 3.8 & - & 2.332 & 0.090 & 0.1085 \\
\hline 13855 & 3.023 & 2 & 3.0 & $\mathrm{~V}_{\mathrm{c}}^{(12,21)}$ & 2.462 & 0.124 & 0.1115 \\
\hline 13894 & 5.478 & 2 & 3.9 & - & 2.359 & 0.093 & 0.1130 \\
\hline 14044 & 9.25874 & 2 & 4.2 & - & 2.431 & 0.106 & 0.1057 \\
\hline 14218 & 6.245 & 2 & 3.2 & $\mathrm{~V}_{\mathrm{c}}^{(2,13)}$ & 2.392 & 0.109 & 0.1024 \\
\hline 14383 & 8.713 & 2 & 3.2 & $\mathrm{~V}_{\mathrm{c}}^{(2,13)}$ & 2.372 & 0.098 & 0.1105 \\
\hline 14385 & 3.78 & 2 & 3.1 & - & 2.360 & 0.097 & 0.1159 \\
\hline 14527 & 6.012 & $3-$ & 2.4 & $\mathrm{~V}_{\mathrm{c}}^{(2,13)}$ & 2.282 & 0.083 & 0.1223 \\
\hline 14568 & 3.523 & 2 & 3.4 & $\mathrm{~V}_{\mathrm{c}}^{(2,13)}$ & 2.393 & 0.089 & 0.1196 \\
\hline 14686 & 4.3283 & 3 & 4.6 & - & 2.334 & 0.090 & 0.1059 \\
\hline 14832 & 8.07 & $2+$ & 4.4 & - & 2.293 & 0.096 & 0.1086 \\
\hline 15032 & 4.405511 & 2 & 3.6 & $\mathrm{~V}_{\mathrm{c}}^{(2,12,13,21)}$ & 2.372 & 0.111 & 0.1096 \\
\hline 15411 & 4.389 & 2 & 3.4 & - & 2.360 & 0.107 & 0.1129 \\
\hline 15634 & 3.002 & 2 & 4.5 & $\mathrm{~V}_{\mathrm{c}}^{(2,13)}$ & 2.348 & 0.086 & 0.1183 \\
\hline 16021 & 13. & 2 & 2.6 & - & 2.335 & 0.102 & 0.1115 \\
\hline 16214 & 6.272 & 2 & 4.1 & - & 2.333 & 0.119 & 0.1136 \\
\hline 16382 & 4.9 & 2 & 2.0 & - & 2.328 & 0.090 & 0.1233 \\
\hline 16462 & 62.366 & 2 & 4.4 & - & 2.465 & 0.117 & 0.1134 \\
\hline 16950 & 3.028 & 2 & 3.3 & - & 2.321 & 0.102 & 0.1270 \\
\hline 16977 & 50.07 & 2 & 4.1 & - & 2.385 & 0.106 & 0.1240 \\
\hline 17035 & 2.899 & 2 & 4.8 & $\mathbf{V}^{(2,4,7,13)}$ & 2.444 & 0.112 & 0.1086 \\
\hline 17079 & 4.18 & $2+$ & 2.8 & $\mathrm{~V}_{\mathrm{c}}^{(2,13)}$ & 2.443 & 0.120 & 0.1081 \\
\hline 17409 & 3.849 & 2 & 2.5 & $\mathrm{~V}_{\mathrm{c}}^{(2,13)}$ & 2.306 & 0.090 & 0.1150 \\
\hline 17469 & 17.172 & 2 & 6.0 & $\mathbf{V}^{(2,4,13)}$ & 2.371 & 0.123 & 0.1163 \\
\hline 17821 & 5.934 & 2 & 2.1 & $\mathrm{~V}_{\mathrm{c}}^{(2,13)}$ & 2.314 & 0.084 & 0.1153 \\
\hline 18527 & 3.3529 & 3 & 3.4 & - & 2.399 & 0.105 & 0.1114 \\
\hline 19298 & 78.54 & 2 & 2.8 & - & 2.392 & 0.114 & 0.1111 \\
\hline 19662 & 5.93 & 3 & 3.2 & - & 2.292 & 0.093 & 0.1193 \\
\hline 19979 & 7.568 & $2+$ & 5.3 & $\mathbf{V}^{(2,4,13)}$ & 2.459 & 0.109 & 0.1053 \\
\hline 20073 & 3.49 & 3 & 2.4 & - & 2.300 & 0.096 & 0.1228 \\
\hline 20300 & 2.62 & $3-$ & 2.7 & $\mathrm{~V}_{\mathrm{c}}^{(2,13)}$ & 2.334 & 0.117 & 0.1190 \\
\hline 20308 & 6.863 & 2 & 3.6 & - & 2.289 & 0.101 & 0.1294 \\
\hline 20537 & 15.626 & 2 & 2.8 & - & 2.311 & 0.100 & 0.1273 \\
\hline 20570 & 4.132 & 2 & 3.6 & - & 2.309 & 0.090 & 0.1148 \\
\hline 21126 & 5.8397 & 3 & 6.2 & - & 2.407 & 0.125 & 0.1079 \\
\hline 21321 & 13.92 & 2 & 3.4 & - & 2.314 & 0.091 & 0.1150 \\
\hline 21341 & 3.502 & 2 & 3.9 & - & 2.391 & 0.096 & 0.1063 \\
\hline 21397 & 5.836 & 2 & 2.1 & - & 2.304 & 0.105 & 0.1172 \\
\hline 21406 & 9.185 & 2 & 2.9 & - & 2.340 & 0.087 & 0.1100 \\
\hline 21485 & 12.26 & 2 & 2.2 & - & 2.352 & 0.084 & 0.1155 \\
\hline 22411 & 5.262 & 2 & 3.8 & - & 2.380 & 0.088 & 0.1074 \\
\hline 22515 & 6.558 & 2 & 2.4 & - & 2.311 & 0.110 & 0.1143 \\
\hline 22666 & 5.218 & 2 & 3.5 & - & 2.477 & 0.120 & 0.1112 \\
\hline 22776 & 3.407 & $3-$ & 3.2 & - & 2.317 & 0.087 & 0.1140 \\
\hline 22908 & 16.375 & 2 & 3.6 & - & 2.312 & 0.104 & 0.1222 \\
\hline 23002 & 6.62 & 2 & 2.3 & - & 2.404 & 0.081 & 0.1067 \\
\hline 23228 & 67.582 & 2 & 2.4 & - & 2.326 & 0.098 & 0.1186 \\
\hline 23521 & 14.464 & 2 & 2.9 & - & 2.285 & 0.091 & 0.1231 \\
\hline
\end{tabular}


D. Oszkiewicz et al.: Spin rates of V-types

Table A.2. continued.

\begin{tabular}{|c|c|c|c|c|c|c|c|}
\hline $\begin{array}{r}\text { Asteroid } \\
\text { No. }\end{array}$ & $\begin{array}{r}\text { Synodic period } \\
.(\mathrm{LCDB})[\mathrm{h}]\end{array}$ & $\begin{array}{l}\text { U-code } \\
(\mathrm{LCDB})\end{array}$ & $\begin{array}{r}\text { Diameter } \\
{[\mathrm{km}]} \\
\end{array}$ & $\begin{array}{l}\text { Class, } \\
\text { references }\end{array}$ & $\begin{array}{c}a \\
{[\mathrm{au}]}\end{array}$ & $e$ & $\sin (i)$ \\
\hline 23588 & 7.197 & 2 & 2.6 & - & 2.408 & 0.097 & 0.1090 \\
\hline 23705 & 82.636 & 2 & 3.8 & - & 2.451 & 0.127 & 0.1121 \\
\hline 23750 & 10.278 & 2 & 2.4 & $\mathrm{~V}_{\mathrm{c}}^{(2,13)}$ & 2.379 & 0.084 & 0.1148 \\
\hline 23758 & 3.12 & 2 & 2.8 & $V_{c}^{(2,13)}$ & 2.337 & 0.094 & 0.1029 \\
\hline 23766 & 2.721 & 3 & 4.5 & - & 2.469 & 0.118 & 0.1138 \\
\hline 23804 & 532.24 & 2 & 2.7 & $\mathrm{~V}_{\mathrm{c}}^{(2,13)}$ & 2.367 & 0.109 & 0.1082 \\
\hline 24115 & 2.635 & 2 & 3.2 & $\mathrm{~V}_{\mathrm{c}}^{(12,21)}$ & 2.402 & 0.112 & 0.1110 \\
\hline 24147 & 5.938 & 2 & 2.0 & - & 2.371 & 0.105 & 0.1099 \\
\hline 24201 & 12.774 & 2 & 2.2 & - & 2.351 & 0.085 & 0.1292 \\
\hline 24304 & 6.139 & 2 & 2.3 & - & 2.328 & 0.091 & 0.1162 \\
\hline 25220 & 8.65 & 2 & 2.7 & $\mathrm{~V}_{\mathrm{c}}^{(12,21)}$ & 2.349 & 0.099 & 0.1231 \\
\hline 25468 & 5.573 & 2 & 4.3 & - & 2.351 & 0.093 & 0.1177 \\
\hline 25469 & 8.57 & 2 & 3.7 & - & 2.323 & 0.098 & 0.1215 \\
\hline 25907 & 6.936 & 2 & 2.7 & - & 2.288 & 0.101 & 0.1258 \\
\hline 26084 & 121.957 & 2 & 2.8 & $V_{c}^{(2,13)}$ & 2.466 & 0.112 & 0.1076 \\
\hline 26230 & 7.318 & 2 & 3.1 & $\mathrm{~V}_{\mathrm{c}}^{(2,13)}$ & 2.456 & 0.120 & 0.1129 \\
\hline 26284 & 7.501 & 2 & 2.9 & $V_{c}^{(2,13)}$ & 2.411 & 0.087 & 0.1211 \\
\hline 26736 & 12.343 & 2 & 2.0 & $\mathrm{~V}_{\mathrm{c}}^{(2,13)}$ & 2.383 & 0.090 & 0.1152 \\
\hline 27018 & 5.733 & 2 & 2.4 & - & 2.354 & 0.097 & 0.1039 \\
\hline 27060 & 5.798 & 2 & 3.5 & $\mathrm{~V}^{(2,13,24)}, \mathrm{S}^{(25,26)}$ & 2.348 & 0.097 & 0.1302 \\
\hline 27377 & 6.182 & 2 & 1.9 & $\mathrm{~V}_{\mathrm{c}}^{(2,13)}$ & 2.328 & 0.093 & 0.1171 \\
\hline 27529 & 4.129 & 2 & 2.8 & $\mathrm{~V}_{\mathrm{c}}^{(2,13)}$ & 2.362 & 0.095 & 0.1154 \\
\hline 27834 & 4.25 & 2 & 3.0 & - & 2.335 & 0.098 & 0.1196 \\
\hline 27880 & 4.998 & 2 & 2.2 & $\mathrm{~V}_{\mathrm{c}}^{(2,13)}$ & 2.378 & 0.118 & 0.1059 \\
\hline 28396 & 12.548 & 2 & 1.9 & - & 2.342 & 0.100 & 0.1168 \\
\hline 28735 & 5.19 & 2 & 3.5 & $V_{c}^{(2,13)}$ & 2.349 & 0.096 & 0.1178 \\
\hline 29140 & 2.99 & 2 & 2.4 & - & 2.380 & 0.105 & 0.1177 \\
\hline 29194 & 6.02 & 2 & 2.7 & - & 2.407 & 0.093 & 0.1041 \\
\hline 29762 & 13.106 & 2 & 3.2 & - & 2.282 & 0.108 & 0.1154 \\
\hline 30329 & 28.4 & $2+$ & 2.9 & $\mathrm{~V}_{\mathrm{c}}^{(12,21)}$ & 2.406 & 0.088 & 0.1028 \\
\hline 30570 & 4.587 & 2 & 3.0 & - & 2.460 & 0.124 & 0.1133 \\
\hline 31108 & 13.237 & 2 & 2.3 & - & 2.309 & 0.085 & 0.1121 \\
\hline 31429 & 36.9 & 2 & 2.5 & - & 2.311 & 0.100 & 0.1247 \\
\hline 31737 & 6.93 & 2 & 3.6 & - & 2.376 & 0.093 & 0.1126 \\
\hline 31888 & 5.13 & 2 & 2.2 & $\mathrm{~V}_{\mathrm{c}}^{(2,13)}$ & 2.353 & 0.106 & 0.1243 \\
\hline 32232 & 5.68 & $2+$ & 4.7 & $V_{c}^{(2,13)}$ & 2.404 & 0.094 & 0.1162 \\
\hline 32522 & 3.62 & 2 & 3.6 & - & 2.376 & 0.089 & 0.1148 \\
\hline 32626 & 6.23 & $3-$ & 3.4 & - & 2.340 & 0.101 & 0.1228 \\
\hline 32678 & 6.985 & 2 & 3.4 & - & 2.366 & 0.116 & 0.1093 \\
\hline 32953 & 4.329 & 2 & 2.4 & - & 2.342 & 0.096 & 0.1180 \\
\hline 33539 & 9.135 & 2 & 2.2 & $\mathrm{~V}_{\mathrm{c}}^{(2,13)}$ & 2.336 & 0.096 & 0.1265 \\
\hline 35821 & 4.931 & 2 & 3.3 & $\mathrm{~V}_{\mathrm{c}}^{(2,13)}$ & 2.482 & 0.109 & 0.1081 \\
\hline 35886 & 3.19 & 2 & 4.0 & - & 2.315 & 0.087 & 0.1261 \\
\hline 35965 & 6.76072 & 2 & 3.2 & $\mathrm{~V}_{\mathrm{c}}^{(2,13,20)}$ & 2.319 & 0.114 & 0.1114 \\
\hline 37088 & 3.359 & 2 & 2.6 & - & 2.363 & 0.108 & 0.1137 \\
\hline 37637 & 5.366 & 2 & 1.8 & $V_{c}^{(2,13)}$ & 2.293 & 0.080 & 0.0917 \\
\hline 37776 & 42.409 & 2 & 1.6 & - & 2.387 & 0.096 & 0.1189 \\
\hline 38140 & 14.476 & 2 & 1.8 & - & 2.276 & 0.093 & 0.1157 \\
\hline 38707 & 6.1509 & $3-$ & 2.3 & - & 2.278 & 0.093 & 0.1123 \\
\hline 38879 & 5.537 & 2 & 2.7 & $\mathrm{~V}_{\mathrm{c}}^{(12,21)}$ & 2.405 & 0.092 & 0.1151 \\
\hline 39051 & 6.4 & 2 & 2.1 & - & 2.309 & 0.098 & 0.1231 \\
\hline 39088 & 5.373 & 2 & 2.4 & - & 2.306 & 0.109 & 0.1138 \\
\hline 39218 & 54.444 & 2 & 3.5 & $\mathrm{~V}_{\mathrm{c}}^{(2,13)}$ & 2.318 & 0.106 & 0.1171 \\
\hline 39494 & 7.353 & 2 & 1.8 & $\mathrm{~V}_{\mathrm{c}}^{(2,13)}$ & 2.300 & 0.106 & 0.1118 \\
\hline 39829 & 5.331 & 2 & 1.6 & - & 2.275 & 0.092 & 0.1123 \\
\hline
\end{tabular}


Table A.2. continued.

\begin{tabular}{|c|c|c|c|c|c|c|c|}
\hline $\begin{array}{r}\text { Asteroid } \\
\text { No. }\end{array}$ & $\begin{array}{r}\text { Synodic period } \\
\text { (LCDB) }[\mathrm{h}]\end{array}$ & $\begin{array}{c}\text { U-code } \\
(\mathrm{LCDB})\end{array}$ & $\begin{array}{r}\text { Diameter } \\
{[\mathrm{km}]} \\
\end{array}$ & $\begin{array}{l}\text { Class, } \\
\text { references }\end{array}$ & $\begin{array}{c}a \\
{[\mathrm{au}]}\end{array}$ & $e$ & $\sin (i)$ \\
\hline 39863 & 9.0488 & 2 & 3.5 & - & 2.304 & 0.112 & 0.1185 \\
\hline 40279 & 6.656 & 2 & 2.1 & - & 2.302 & 0.097 & 0.1176 \\
\hline 40370 & 7.38 & $3-$ & 1.6 & - & 2.292 & 0.079 & 0.1127 \\
\hline 40408 & 9.217 & 2 & 2.0 & - & 2.341 & 0.093 & 0.1259 \\
\hline 40453 & 17.442 & 2 & 2.5 & - & 2.323 & 0.085 & 0.1141 \\
\hline 40574 & 5.346 & 2 & 1.7 & - & 2.336 & 0.099 & 0.1068 \\
\hline 41789 & 8.932 & 2 & 3.5 & - & 2.296 & 0.111 & 0.1194 \\
\hline 42644 & 16.0 & $2-$ & 3.9 & $\mathrm{~V}_{\mathrm{c}}^{(12,21)}$ & 2.367 & 0.103 & 0.1161 \\
\hline 42653 & 5.495 & 2 & 2.2 & - & 2.299 & 0.104 & 0.1169 \\
\hline 42655 & 5.168 & 2 & 2.8 & - & 2.329 & 0.105 & 0.1092 \\
\hline 42866 & 42.227 & 2 & 2.7 & - & 2.354 & 0.104 & 0.1201 \\
\hline 42915 & 5.504 & 2 & 2.1 & - & 2.286 & 0.081 & 0.1102 \\
\hline 42953 & 2.67 & 2 & 2.3 & - & 2.318 & 0.115 & 0.1104 \\
\hline 43463 & 9.697 & 2 & 1.6 & - & 2.320 & 0.099 & 0.1168 \\
\hline 43464 & 101.649 & 2 & 2.0 & - & 2.297 & 0.093 & 0.1171 \\
\hline 43492 & 5.08 & $2+$ & 1.6 & - & 2.313 & 0.089 & 0.1266 \\
\hline 43941 & 5.374 & 2 & 2.7 & - & 2.303 & 0.092 & 0.1167 \\
\hline 44569 & 5.514 & 2 & 2.0 & $\mathrm{~V}_{\mathrm{c}}^{(21)}$ & 2.275 & 0.095 & 0.1271 \\
\hline 44681 & 17.337 & 2 & 1.7 & $\mathrm{~V}_{\mathrm{c}}^{(2,13)}$ & 2.282 & 0.106 & 0.1164 \\
\hline 44864 & 9.121 & 2 & 1.9 & $\mathrm{~V}_{\mathrm{c}}^{(2,13)}$ & 2.368 & 0.082 & 0.1034 \\
\hline 44872 & 5.068 & 2 & 2.3 & - & 2.324 & 0.096 & 0.1116 \\
\hline 45041 & 4.66 & 3 & 2.1 & - & 2.342 & 0.101 & 0.1213 \\
\hline 45052 & 2.721 & 2 & 1.9 & - & 2.330 & 0.105 & 0.1154 \\
\hline 45073 & 3.16 & $3-$ & 3.0 & $\mathrm{~V}_{\mathrm{c}}^{(2,13)}$ & 2.334 & 0.123 & 0.1151 \\
\hline 45160 & 4.104 & 2 & 2.0 & - & 2.311 & 0.111 & 0.1177 \\
\hline 45270 & 7.851 & 2 & 2.8 & - & 2.393 & 0.089 & 0.1184 \\
\hline 45986 & 5.85 & $2-$ & 2.1 & - & 2.340 & 0.095 & 0.1158 \\
\hline 46784 & 19. & $2-$ & 2.0 & - & 2.252 & 0.104 & 0.1174 \\
\hline 47229 & 47.168 & 2 & 2.0 & - & 2.305 & 0.089 & 0.1206 \\
\hline 47271 & 6.902 & 2 & 3.0 & - & 2.379 & 0.117 & 0.1189 \\
\hline 47332 & 3.992 & 2 & 2.4 & - & 2.370 & 0.103 & 0.1078 \\
\hline 47369 & 2.55 & 2 & 4.3 & - & 2.390 & 0.119 & 0.1075 \\
\hline 47406 & 5.838 & 2 & 1.8 & - & 2.392 & 0.094 & 0.1016 \\
\hline 47528 & 6.44 & $2+$ & 2.3 & - & 2.416 & 0.091 & 0.1011 \\
\hline 48094 & 11.613 & 2 & 1.9 & - & 2.318 & 0.092 & 0.1210 \\
\hline 48120 & 78.226 & 2 & 2.1 & - & 2.391 & 0.101 & 0.1159 \\
\hline 48134 & 5.778 & 2 & 2.3 & - & 2.360 & 0.106 & 0.1191 \\
\hline 48190 & 2.4 & 2 & 1.9 & - & 2.275 & 0.082 & 0.1053 \\
\hline 48376 & 534.962 & 2 & 2.7 & - & 2.345 & 0.107 & 0.1017 \\
\hline 48507 & 6.9 & 2 & 3.0 & - & 2.344 & 0.096 & 0.1191 \\
\hline 48644 & 8.28 & $2-$ & 2.6 & $\mathrm{~V}_{\mathrm{c}}^{(12,21)}$ & 2.436 & 0.099 & 0.1076 \\
\hline 48734 & 6.446 & 2 & 2.2 & $\mathrm{~V}_{\mathrm{c}}^{(12,21)}$ & 2.281 & 0.080 & 0.1136 \\
\hline 48748 & 7.018 & 2 & 2.1 & - & 2.293 & 0.110 & 0.1196 \\
\hline 48907 & 13.896 & 2 & 1.9 & - & 2.347 & 0.096 & 0.1056 \\
\hline 48935 & 5.363 & 2 & 2.4 & $\mathrm{~V}_{\mathrm{c}}^{(2,13)}$ & 2.297 & 0.093 & 0.1200 \\
\hline 48966 & 9.203 & 2 & 2.6 & - & 2.392 & 0.092 & 0.1260 \\
\hline 48981 & 6.979 & 2 & 3.7 & - & 2.338 & 0.107 & 0.1114 \\
\hline 48997 & 6.55436 & 2 & 3.5 & - & 2.305 & 0.112 & 0.1183 \\
\hline 48999 & 7.044 & 2 & 2.5 & - & 2.346 & 0.095 & 0.1145 \\
\hline 49039 & 72. & 2 & 3.1 & - & 2.349 & 0.097 & 0.1185 \\
\hline 50025 & 11.003 & 2 & 2.3 & $\mathrm{~V}_{\mathrm{c}}^{(2,13)}$ & 2.365 & 0.103 & 0.1088 \\
\hline 50138 & 11.38 & 2 & 2.0 & - & 2.379 & 0.113 & 0.1114 \\
\hline 50179 & 6.066 & 2 & 1.6 & - & 2.350 & 0.092 & 0.1061 \\
\hline 50196 & 10.166 & 2 & 2.9 & $\mathrm{~V}_{\mathrm{c}}^{(2,13)}$ & 2.310 & 0.097 & 0.1240 \\
\hline 50329 & 7.06 & 2 & 2.5 & - & 2.340 & 0.092 & 0.1188 \\
\hline 51609 & 6.767 & 3 & 2.0 & - & 2.309 & 0.107 & 0.1118 \\
\hline 52852 & 5.432 & $3-$ & 2.5 & - & 2.263 & 0.097 & 0.1211 \\
\hline
\end{tabular}


D. Oszkiewicz et al.: Spin rates of V-types

Table A.2. continued.

\begin{tabular}{|c|c|c|c|c|c|c|c|}
\hline $\begin{array}{r}\text { Asteroid } \\
\text { No. }\end{array}$ & $\begin{array}{r}\text { Synodic period } \\
.(\mathrm{LCDB})(\mathrm{h})\end{array}$ & $\begin{array}{l}\text { U-code } \\
\text { (LCDB) }\end{array}$ & $\begin{array}{r}\text { Diameter } \\
(\mathrm{km}) \\
\end{array}$ & $\begin{array}{l}\text { Class, } \\
\text { references }\end{array}$ & $\begin{array}{c}a \\
(\mathrm{au})\end{array}$ & $e$ & $\sin (i)$ \\
\hline 53515 & 8.652 & 2 & 2.8 & $\mathrm{~V}_{\mathrm{c}}^{(2,13)}$ & 2.327 & 0.120 & 0.1176 \\
\hline 53632 & 9.055 & 2 & 1.9 & $\mathrm{~V}_{\mathrm{c}}^{(2,13)}$ & 2.393 & 0.099 & 0.1107 \\
\hline 53734 & 5.05 & 2 & 2.7 & $\mathrm{~V}_{\mathrm{c}}^{(2,12,13,21)}$ & 2.335 & 0.120 & 0.1161 \\
\hline 53930 & 10.593 & 2 & 1.7 & - & 2.448 & 0.096 & 0.1050 \\
\hline 54076 & 4.95 & 2 & 2.0 & - & 2.377 & 0.097 & 0.1095 \\
\hline 55293 & 4.62 & 2 & 1.6 & - & 2.350 & 0.103 & 0.1147 \\
\hline 55459 & 11.308 & 2 & 2.0 & - & 2.447 & 0.114 & 0.1118 \\
\hline 56394 & 9.32 & $2+$ & 1.9 & - & 2.452 & 0.097 & 0.1075 \\
\hline 57478 & 5.887 & $3-$ & 2.5 & - & 2.342 & 0.096 & 0.1220 \\
\hline 58049 & 5.376 & 2 & 1.2 & - & 2.343 & 0.086 & 0.1178 \\
\hline 58658 & 4.95 & 2 & 2.1 & - & 2.436 & 0.098 & 0.1125 \\
\hline 59291 & 2.8868 & $3-$ & 3.1 & - & 2.286 & 0.095 & 0.1293 \\
\hline 59345 & 6.19 & 2 & 3.1 & - & 2.428 & 0.103 & 0.1083 \\
\hline 59489 & 5.455 & 2 & 3.4 & - & 2.398 & 0.116 & 0.1132 \\
\hline 60326 & 18.993 & 2 & 1.8 & - & 2.328 & 0.097 & 0.1139 \\
\hline 60507 & 9.411 & 2 & 2.2 & - & 2.355 & 0.092 & 0.1042 \\
\hline 61036 & 6.617 & 2 & 1.7 & - & 2.353 & 0.094 & 0.1042 \\
\hline 63264 & 7.74 & $2-$ & 1.6 & - & 2.323 & 0.095 & 0.1276 \\
\hline 63702 & 4.66 & 2 & 2.8 & - & 2.473 & 0.106 & 0.1096 \\
\hline 64599 & 9.89378 & 2 & 2.3 & - & 2.329 & 0.100 & 0.1203 \\
\hline 65068 & 8.763 & 2 & 1.8 & $\mathrm{~V}_{\mathrm{c}}^{(2,12,13,21)}$ & 2.290 & 0.107 & 0.1242 \\
\hline 65242 & 5.168 & 2 & 2.2 & - & 2.389 & 0.079 & 0.1212 \\
\hline 65345 & 14.148 & 2 & 1.8 & - & 2.417 & 0.094 & 0.1094 \\
\hline 65719 & 7.18 & 2 & 2.0 & $\mathrm{~V}_{\mathrm{c}}^{(2,13)}$ & 2.308 & 0.092 & 0.1181 \\
\hline 66189 & 7.88 & 3 & 1.7 & - & 2.314 & 0.116 & 0.1101 \\
\hline 67847 & 2.473 & 2 & 1.5 & - & 2.265 & 0.111 & 0.1084 \\
\hline 67909 & 13.567 & 2 & 2.8 & - & 2.287 & 0.091 & 0.1265 \\
\hline 69076 & 4.829 & 2 & 1.8 & $\mathrm{~V}_{\mathrm{c}}^{(2,13)}$ & 2.332 & 0.091 & 0.1268 \\
\hline 69253 & 10.967 & 2 & 1.2 & - & 2.286 & 0.100 & 0.1259 \\
\hline 69267 & 6.44372 & 2 & 3.4 & - & 2.359 & 0.104 & 0.1088 \\
\hline 69595 & 6.67 & $3-$ & 1.7 & - & 2.297 & 0.096 & 0.1226 \\
\hline 69596 & 7.02 & 2 & 2.2 & $\mathrm{~V}_{\mathrm{c}}^{(2,13)}$ & 2.314 & 0.089 & 0.1201 \\
\hline 70085 & 4.95 & 2 & 2.0 & - & 2.273 & 0.088 & 0.1146 \\
\hline 70301 & 9.561 & 2 & 2.2 & - & 2.360 & 0.097 & 0.1204 \\
\hline 70478 & 12.125 & 2 & 2.3 & - & 2.336 & 0.084 & 0.1190 \\
\hline 74219 & 5.7 & $2-$ & 5.4 & - & 2.464 & 0.118 & 0.1144 \\
\hline 74639 & 5.08 & 2 & 1.5 & - & 2.303 & 0.090 & 0.0999 \\
\hline 74885 & 20.87 & 2 & 1.1 & - & 2.314 & 0.094 & 0.1182 \\
\hline 75011 & 2.8 & 2 & 2.5 & $\mathrm{~V}_{\mathrm{c}}^{(2,13)}$ & 2.324 & 0.096 & 0.1162 \\
\hline 75403 & 7.139 & 2 & 1.9 & - & 2.436 & 0.113 & 0.1108 \\
\hline 75423 & 2.552 & 2 & 2.2 & - & 2.386 & 0.085 & 0.1084 \\
\hline 75426 & 7.45 & 2 & 2.7 & - & 2.392 & 0.093 & 0.1175 \\
\hline 75605 & 4.68 & 2 & 2.0 & - & 2.410 & 0.115 & 0.1100 \\
\hline 76898 & 14.138 & 2 & 2.1 & - & 2.322 & 0.100 & 0.1213 \\
\hline 77186 & 32.112 & 2 & 2.0 & $\mathrm{~V}_{\mathrm{c}}^{(2,13)}$ & 2.356 & 0.093 & 0.1172 \\
\hline 77357 & 2.6 & $2-$ & 2.4 & - & 2.366 & 0.086 & 0.1059 \\
\hline 77590 & 5.892 & 2 & 2.1 & $\mathrm{~V}_{\mathrm{c}}^{(2,12,13,21)}$ & 2.402 & 0.106 & 0.1062 \\
\hline 79559 & 62.854 & 2 & 2.9 & - & 2.415 & 0.116 & 0.1095 \\
\hline 79621 & 3.512 & 2 & 2.1 & - & 2.398 & 0.105 & 0.1162 \\
\hline 79639 & 6.026 & 2 & 3.3 & - & 2.320 & 0.098 & 0.1269 \\
\hline 79704 & 6.4 & $2+$ & 2.0 & - & 2.393 & 0.100 & 0.1106 \\
\hline 79738 & 7.28 & 2 & 2.0 & - & 2.408 & 0.105 & 0.1276 \\
\hline 80180 & 5.71 & 2 & 1.6 & - & 2.309 & 0.104 & 0.1097 \\
\hline 80186 & 41.094 & 2 & 1.1 & - & 2.314 & 0.120 & 0.1091 \\
\hline 80234 & 4.32 & $2+$ & 1.4 & - & 2.294 & 0.096 & 0.1230 \\
\hline 80371 & 5.128 & 2 & 1.6 & - & 2.317 & 0.092 & 0.1059 \\
\hline
\end{tabular}


Table A.2. continued.

\begin{tabular}{|c|c|c|c|c|c|c|c|}
\hline $\begin{array}{r}\text { Asteroid } \\
\text { No. }\end{array}$ & $\begin{array}{r}\text { Synodic period } \\
.(\mathrm{LCDB})(\mathrm{h})\end{array}$ & $\begin{array}{l}\text { U-code } \\
(\mathrm{LCDB})\end{array}$ & $\begin{array}{r}\text { Diameter } \\
(\mathrm{km}) \\
\end{array}$ & $\begin{array}{l}\text { Class, } \\
\text { references }\end{array}$ & $\begin{array}{c}a \\
(\mathrm{au})\end{array}$ & $e$ & $\sin (i)$ \\
\hline 80438 & 4.682 & 2 & 1.4 & - & 2.344 & 0.086 & 0.1161 \\
\hline 80623 & 5.057 & $2+$ & 1.6 & - & 2.324 & 0.121 & 0.1139 \\
\hline 80678 & 5.066 & 2 & 2.1 & - & 2.379 & 0.104 & 0.1055 \\
\hline 80704 & 10.535 & 2 & 1.7 & - & 2.256 & 0.095 & 0.1103 \\
\hline 80775 & 5.362 & 2 & 1.6 & - & 2.394 & 0.099 & 0.1076 \\
\hline 81263 & 6.3 & $3-$ & 2.0 & $\mathrm{~V}_{\mathrm{c}}^{(2,13)}$ & 2.406 & 0.110 & 0.1091 \\
\hline 82660 & 5.631 & 2 & 2.0 & - & 2.461 & 0.128 & 0.1105 \\
\hline 84042 & 8.07 & 2 & 1.3 & - & 2.273 & 0.098 & 0.1210 \\
\hline 84668 & 6.67 & $2+$ & 2.2 & - & 2.461 & 0.121 & 0.1063 \\
\hline 85959 & 89.669 & 2 & 2.1 & - & 2.268 & 0.090 & 0.1169 \\
\hline 86492 & 5.466 & 2 & 1.5 & $\mathrm{~V}_{\mathrm{c}}^{(2,13)}$ & 2.325 & 0.107 & 0.1193 \\
\hline 88352 & 7.01 & $2-$ & 2.3 & - & 2.385 & 0.099 & 0.1124 \\
\hline 88367 & 3.962 & 2 & 2.1 & - & 2.389 & 0.087 & 0.1134 \\
\hline 88531 & 2.9 & 2 & 1.4 & - & 2.370 & 0.093 & 0.1106 \\
\hline 88622 & 4.524 & 2 & 2.2 & - & 2.381 & 0.105 & 0.1038 \\
\hline 89410 & 10.895 & 2 & 2.4 & - & 2.443 & 0.104 & 0.1163 \\
\hline 89413 & 7.876 & 2 & 1.8 & - & 2.433 & 0.090 & 0.1177 \\
\hline 89736 & 10.699 & 2 & 2.6 & - & 2.462 & 0.123 & 0.1119 \\
\hline 96817 & 5.631 & 2 & 2.3 & - & 2.462 & 0.115 & 0.1111 \\
\hline 97674 & 8.161 & 2 & 2.1 & - & 2.478 & 0.103 & 0.1074 \\
\hline 98384 & 67.293 & 2 & 1.5 & - & 2.353 & 0.101 & 0.1208 \\
\hline 98603 & 8.052 & 2 & 1.7 & - & 2.295 & 0.112 & 0.1159 \\
\hline 99656 & 5.339 & 2 & 1.8 & - & 2.404 & 0.085 & 0.1161 \\
\hline 99742 & 4.663 & 2 & 1.3 & - & 2.272 & 0.100 & 0.1240 \\
\hline 101924 & 4.231 & 2 & 1.5 & - & 2.280 & 0.113 & 0.1097 \\
\hline 102112 & 63.867 & 2 & 1.4 & - & 2.271 & 0.105 & 0.1076 \\
\hline 102380 & 63.867 & 2 & 1.4 & - & 2.300 & 0.110 & 0.1112 \\
\hline 103071 & 7.399 & 2 & 1.1 & - & 2.393 & 0.097 & 0.1273 \\
\hline 103132 & 2.51 & $2+$ & 2.2 & $\mathrm{~V}_{\mathrm{c}}^{(12,21)}$ & 2.453 & 0.118 & 0.1122 \\
\hline 104182 & 195.786 & 2 & 1.6 & - & 2.409 & 0.105 & 0.1110 \\
\hline 107026 & 2.789 & 2 & 1.3 & - & 2.295 & 0.089 & 0.1198 \\
\hline 107587 & 4.165 & 2 & 1.5 & - & 2.319 & 0.096 & 0.1183 \\
\hline 107750 & 5.605 & 2 & 1.4 & - & 2.338 & 0.080 & 0.1131 \\
\hline 108438 & 17.466 & 2 & 1.4 & - & 2.281 & 0.086 & 0.1216 \\
\hline 109187 & 19.737 & 2 & 1.4 & - & 2.324 & 0.089 & 0.1257 \\
\hline 114146 & 7.626 & 2 & 1.5 & - & 2.405 & 0.095 & 0.1164 \\
\hline 115358 & 4.85 & $2-$ & 1.5 & - & 2.376 & 0.091 & 0.1248 \\
\hline 115758 & 42.577 & 2 & 1.5 & - & 2.298 & 0.093 & 0.1205 \\
\hline 116588 & 5.61 & 2 & 1.2 & - & 2.259 & 0.095 & 0.1177 \\
\hline 117247 & 50.985 & 2 & 1.6 & - & 2.291 & 0.101 & 0.1244 \\
\hline 118293 & 16.235 & 2 & 1.3 & $\mathrm{~V}_{\mathrm{c}}^{(2,13)}$ & 2.325 & 0.104 & 0.1074 \\
\hline 120019 & 6.266 & 2 & 1.7 & - & 2.334 & 0.106 & 0.1117 \\
\hline 124297 & 5.78 & 2 & 1.6 & $\mathrm{~V}_{\mathrm{c}}^{(2,13)}$ & 2.383 & 0.093 & 0.1036 \\
\hline 124548 & 6.498 & 2 & 1.7 & $\mathrm{~V}_{\mathrm{c}}^{(2,13)}$ & 2.359 & 0.091 & 0.1273 \\
\hline 125091 & 4.45 & $2+$ & 2.0 & - & 2.314 & 0.097 & 0.1254 \\
\hline 125390 & 9.14 & 2 & 1.5 & $\mathrm{~V}_{\mathrm{c}}^{(21)}$ & 2.377 & 0.090 & 0.1159 \\
\hline 129334 & 5.16 & 2 & 0.9 & - & 2.323 & 0.097 & 0.1212 \\
\hline 130682 & 5.046 & 2 & 2.5 & - & 2.393 & 0.097 & 0.1194 \\
\hline 130753 & 53.595 & 2 & 1.7 & - & 2.404 & 0.093 & 0.1074 \\
\hline 130820 & 12.98 & 2 & 1.4 & - & 2.394 & 0.100 & 0.1019 \\
\hline 134702 & 9.007 & 2 & 0.9 & - & 2.395 & 0.109 & 0.1097 \\
\hline 136785 & 37.614 & 2 & 1.3 & - & 2.287 & 0.098 & 0.1259 \\
\hline 137280 & 62.323 & 2 & 1.1 & - & 2.279 & 0.083 & 0.1168 \\
\hline 137366 & 5.522 & 2 & 1.3 & - & 2.300 & 0.107 & 0.1264 \\
\hline 137736 & 67.796 & 2 & 1.4 & - & 2.398 & 0.112 & 0.1047 \\
\hline 139201 & 7.719 & 2 & 1.3 & - & 2.343 & 0.099 & 0.1296 \\
\hline 139312 & 4.003 & 2 & 1.3 & - & 2.384 & 0.100 & 0.1172 \\
\hline
\end{tabular}


D. Oszkiewicz et al.: Spin rates of V-types

Table A.2. continued.

\begin{tabular}{|c|c|c|c|c|c|c|c|}
\hline $\begin{array}{r}\text { Asteroid } \\
\text { No. }\end{array}$ & $\begin{array}{r}\text { Synodic period } \\
.(\mathrm{LCDB})(\mathrm{h})\end{array}$ & $\begin{array}{l}\text { U-code } \\
(\mathrm{LCDB})\end{array}$ & $\begin{array}{r}\text { Diameter } \\
(\mathrm{km}) \\
\end{array}$ & $\begin{array}{l}\text { Class, } \\
\text { references }\end{array}$ & $\begin{array}{c}a \\
(\mathrm{au})\end{array}$ & $e$ & $\sin (i)$ \\
\hline 142061 & 62.558 & 2 & 1.1 & - & 2.324 & 0.108 & 0.1171 \\
\hline 143946 & 7.695 & 2 & 1.2 & $\mathrm{~V}_{\mathrm{c}}^{(2,13)}$ & 2.302 & 0.096 & 0.1102 \\
\hline 143950 & 17.105 & 2 & 1.3 & - & 2.387 & 0.108 & 0.1137 \\
\hline 144033 & 4.368 & 2 & 1.4 & $\mathrm{~V}_{\mathrm{c}}^{(2,13)}$ & 2.418 & 0.098 & 0.1114 \\
\hline 144067 & 5.49 & 2 & 1.4 & - & 2.478 & 0.102 & 0.1074 \\
\hline 144564 & 138.112 & 2 & 1.8 & - & 2.333 & 0.114 & 0.1052 \\
\hline 144974 & 246.672 & 2 & 0.9 & - & 2.274 & 0.083 & 0.1190 \\
\hline 145092 & 20.051 & 2 & 1.2 & - & 2.310 & 0.096 & 0.1219 \\
\hline 147037 & 4.234 & 2 & 1.6 & - & 2.354 & 0.094 & 0.1188 \\
\hline 147887 & 4.6566 & 3 & 1.6 & - & 2.351 & 0.086 & 0.1205 \\
\hline 149379 & 8.449 & 2 & 1.2 & - & 2.400 & 0.103 & 0.1018 \\
\hline 149385 & 3.316 & 2 & 1.6 & - & 2.354 & 0.084 & 0.1170 \\
\hline 150080 & 4.728 & 2 & 1.0 & - & 2.334 & 0.086 & 0.1160 \\
\hline 150789 & 5.242 & 2 & 1.2 & - & 2.330 & 0.098 & 0.1249 \\
\hline 152212 & 7.27 & 2 & 1.2 & - & 2.336 & 0.104 & 0.1122 \\
\hline 153672 & 28.24 & 2 & 1.5 & - & 2.432 & 0.103 & 0.1129 \\
\hline 161772 & 7.194 & 2 & 1.1 & $\mathrm{~V}_{\mathrm{c}}^{(2,13)}$ & 2.368 & 0.084 & 0.1283 \\
\hline 162577 & 6.085 & 2 & 1.2 & - & 2.343 & 0.090 & 0.1064 \\
\hline 167138 & 4.206 & 2 & 1.3 & - & 2.298 & 0.109 & 0.1164 \\
\hline 171400 & 4.852 & 2 & 1.8 & - & 2.411 & 0.125 & 0.1122 \\
\hline 172473 & 5.360 & 2 & 1.0 & - & 2.288 & 0.106 & 0.1033 \\
\hline 172601 & 39.971 & 2 & 1.0 & - & 2.320 & 0.086 & 0.1030 \\
\hline 173255 & 4.638 & 2 & 1.4 & - & 2.287 & 0.098 & 0.1218 \\
\hline 174606 & 5.666 & 2 & 1.3 & - & 2.270 & 0.098 & 0.1227 \\
\hline 174655 & 4.638 & 2 & 1.4 & - & 2.304 & 0.095 & 0.1166 \\
\hline 174830 & 42.158 & 2 & 1.6 & - & 2.403 & 0.126 & 0.1122 \\
\hline 174837 & 42.97 & 2 & 1.4 & - & 2.386 & 0.097 & 0.1176 \\
\hline 175809 & 202.127 & 2 & 1.0 & - & 2.291 & 0.107 & 0.1203 \\
\hline 175856 & 4.96 & 2 & 1.3 & - & 2.321 & 0.096 & 0.1172 \\
\hline 176111 & 6.29 & 2 & 1.2 & - & 2.332 & 0.097 & 0.1144 \\
\hline 176139 & 5.13 & 2 & 0.9 & $\mathrm{~V}_{\mathrm{c}}^{(2,13)}$ & 2.313 & 0.105 & 0.1096 \\
\hline 176829 & 14.596 & 2 & 1.3 & - & 2.453 & 0.103 & 0.1196 \\
\hline 177302 & 7.735 & 2 & 1.3 & - & 2.399 & 0.105 & 0.1271 \\
\hline 179604 & 31.426 & 2 & 0.9 & - & 2.344 & 0.115 & 0.1099 \\
\hline 180511 & 13.288 & 2 & 1.5 & - & 2.450 & 0.113 & 0.1155 \\
\hline 183303 & 6.006 & 2 & 1.4 & - & 2.390 & 0.120 & 0.1054 \\
\hline 183708 & 6.519 & 2 & 1.0 & - & 2.294 & 0.087 & 0.1125 \\
\hline 184483 & 6.998 & 2 & 1.4 & - & 2.422 & 0.097 & 0.1066 \\
\hline 186652 & 5.22 & 2 & 0.9 & - & 2.279 & 0.084 & 0.1033 \\
\hline 189740 & 9.32 & 2 & 1.3 & - & 2.436 & 0.116 & 0.1136 \\
\hline 194458 & 6.52 & 2 & 1.2 & - & 2.344 & 0.090 & 0.1086 \\
\hline 194912 & 12.68 & 2 & 1.9 & - & 2.381 & 0.095 & 0.1120 \\
\hline 209060 & 4.14 & $3-$ & 1.7 & - & 2.390 & 0.086 & 0.1155 \\
\hline 220143 & 3.502 & $2+$ & 1.3 & - & 2.324 & 0.088 & 0.1110 \\
\hline 220205 & 9.835 & 2 & 1.1 & - & 2.404 & 0.089 & 0.1100 \\
\hline 221153 & 5.422 & 2 & 1.0 & - & 2.480 & 0.124 & 0.1164 \\
\hline 223121 & 6.003 & 2 & 1.1 & $\mathrm{~V}_{\mathrm{c}}^{(2,13)}$ & 2.305 & 0.085 & 0.1192 \\
\hline 226642 & 4.962 & 2 & 1.4 & $\mathrm{~V}_{\mathrm{c}}^{(2,13)}$ & 2.342 & 0.097 & 0.1185 \\
\hline 230162 & 5.78 & 2 & 1.2 & - & 2.378 & 0.093 & 0.1082 \\
\hline 253531 & 10.158 & 2 & 1.2 & - & 2.299 & 0.096 & 0.1333 \\
\hline 253725 & 6.497 & 2 & 1.0 & - & 2.344 & 0.094 & 0.1142 \\
\hline 253734 & 6.182 & 2 & 0.9 & - & 2.330 & 0.104 & 0.1115 \\
\hline 255623 & 59.226 & 2 & 1.1 & - & 2.406 & 0.106 & 0.1096 \\
\hline 257325 & 4.62 & $2+$ & 1.0 & - & 2.439 & 0.101 & 0.1175 \\
\hline 257631 & 13.17 & 2 & 0.7 & - & 2.301 & 0.119 & 0.1094 \\
\hline 259709 & 9.859 & 2 & 1.2 & $\mathrm{~V}_{\mathrm{c}}^{(2,13)}$ & 2.293 & 0.101 & 0.1244 \\
\hline
\end{tabular}


Table A.2. continued.

\begin{tabular}{rccclccc}
\hline \hline $\begin{array}{r}\text { Asteroid } \\
\text { No. }\end{array}$ & $\begin{array}{c}\text { Synodic period } \\
\text { (LCDB) }(\mathrm{h})\end{array}$ & $\begin{array}{r}\text { U-code } \\
(\text { LCDB })\end{array}$ & $\begin{array}{r}\text { Diameter } \\
(\mathrm{km})\end{array}$ & $\begin{array}{l}\text { Class, } \\
\text { references }\end{array}$ & $\begin{array}{c}a \\
(\mathrm{au})\end{array}$ & $e$ & $\sin (i)$ \\
\hline 262418 & 5.887 & 2 & 1.1 & - & 2.334 & 0.100 & 0.1246 \\
264331 & 5.85 & 2 & 1.0 & - & 2.420 & 0.114 & 0.1128 \\
270739 & 5.462 & 2 & 0.9 & - & 2.340 & 0.097 & 0.1184 \\
272214 & 20.278 & 2 & 1.1 & $\mathrm{~V}_{\mathrm{c}}^{(2,13)}$ & 2.299 & 0.105 & 0.1218 \\
273498 & 9.1 & 2 & 1.4 & - & 2.448 & 0.101 & 0.1117 \\
279536 & 9.189 & 2 & 1.3 & - & 2.406 & 0.091 & 0.1114 \\
283252 & 8.28 & 2 & 1.2 & - & 2.425 & 0.100 & 0.1159 \\
294796 & 8.833 & 2 & 1.0 & - & 2.334 & 0.094 & 0.1238 \\
308335 & 5.89 & 2 & 1.1 & - & 2.283 & 0.085 & 0.1151 \\
316495 & 5.186 & 2 & 0.8 & - & 2.261 & 0.097 & 0.1252 \\
323324 & 4.389 & 2 & 0.9 & - & 2.323 & 0.109 & 0.1104 \\
332477 & 13.52 & $2-$ & 1.0 & - & 2.376 & 0.117 & 0.1191 \\
333887 & 3.914 & 2 & 1.0 & $\mathrm{~V}_{\mathrm{c}}^{(2,13)}$ & 2.307 & 0.086 & 0.1192 \\
334150 & 5.294 & 2 & 1.1 & - & 2.377 & 0.095 & 0.1195 \\
337824 & 2.548 & 2 & 1.0 & - & 2.420 & 0.089 & 0.1094 \\
339771 & 4.95 & 2 & 0.9 & - & 2.280 & 0.097 & 0.1180 \\
339899 & 8.47 & 2 & 1.5 & - & 2.327 & 0.108 & 0.1039 \\
349755 & 8.47 & $2-$ & 1.5 & - & 2.452 & 0.111 & 0.1091 \\
387135 & 4.059 & 2 & 1.3 & - & 2.445 & 0.101 & 0.1075 \\
390500 & 3.23 & 2 & 0.8 & - & 2.281 & 0.103 & 0.1092 \\
\hline
\end{tabular}

Table A.3. As in Table A.2, but for the fugitive population.

\begin{tabular}{rccrllll}
\hline \hline $\begin{array}{r}\text { Asteroid } \\
\text { No. }\end{array}$ & $\begin{array}{c}\text { Synodic period } \\
\text {.(LCDB) }(\mathrm{h})\end{array}$ & $\begin{array}{c}\text { U-code } \\
(\mathrm{LCDB})\end{array}$ & $\begin{array}{r}\text { Diameter } \\
(\mathrm{km})\end{array}$ & $\begin{array}{l}\text { Class, } \\
\text { references }\end{array}$ & $\begin{array}{c}a \\
(\mathrm{au})\end{array}$ & $e$ & $\sin (i)$ \\
\hline 809 & 15.4142 & 3 & 9.5 & $\mathrm{~V}^{(1,4,15,17,19)}$ & 2.283 & 0.145 & 0.1179 \\
956 & 16.492 & 3 & 10.5 & $\mathrm{~V}^{(1,4,15,17,19)}$ & 2.298 & 0.158 & 0.1119 \\
2049 & 8.91 & 2 & 2.5 & $\mathrm{~V}_{\mathrm{c}}^{(2,14)}$ & 1.949 & 0.074 & 0.4109 \\
$\mathbf{2 4 8 6}$ & 4.4518 & 3 & 7.9 & $\mathrm{~V}^{(8,10,13,18)}$ & 2.268 & 0.122 & 0.1410 \\
2579 & 3.63599 & 3 & 4.6 & $\mathrm{~V}^{(1,5,15,16,17)}$ & 2.210 & 0.082 & 0.1054 \\
2912 & 5.71076 & 3 & 6.5 & $\mathrm{~V}^{(1,15,16,17)}$ & 2.289 & 0.118 & 0.1186 \\
3307 & 4.902 & $3-$ & 3.6 & $\mathrm{~V}^{(1,15)}$ & 2.259 & 0.100 & 0.1212 \\
3850 & 2.4289 & 3 & 4.2 & $\mathrm{~V}^{(1,15)}$ & 2.235 & 0.105 & 0.0814 \\
4692 & 2.736 & $2+$ & 4.9 & $\mathrm{~V}_{\mathrm{c}}^{(2,14)}$ & 2.256 & 0.122 & 0.0505 \\
$\mathbf{5 0 3 7}$ & 2.8290 & - & 5.7 & $\mathrm{QV}_{p}^{(2,14)}, \mathrm{V}_{\mathrm{c}}^{(20)}$ & 2.273 & 0.143 & 0.1282 \\
$\mathbf{5 2 3 5}$ & 2.4524 & - & 6.7 & $\mathrm{~V}^{(2,12,14)}$ & 2.297 & 0.120 & 0.0961 \\
5525 & 14.088 & $3-$ & 5.3 & $\mathrm{~V}^{(2,7,14)}$ & 2.221 & 0.081 & 0.1279 \\
5560 & 7.732 & $3-$ & 4.7 & $\mathrm{~V}^{(2,7,14)}$ & 2.286 & 0.118 & 0.0852 \\
5754 & 8.9021 & 3 & 6.3 & $\mathrm{~V}_{\mathrm{c}}^{(2,11,14)}$ & 2.267 & 0.091 & 0.0843 \\
5952 & 4.512546 & 3 & 4.9 & $\mathrm{~V}^{(2,11,13,14,18)}$ & 2.270 & 0.141 & 0.0796 \\
6406 & 6.819 & 3 & 4.1 & $\mathrm{~V}^{(8,9,13,18)}$ & 2.276 & 0.124 & 0.1336 \\
$\mathbf{6 8 1 9}$ & 2.7249 & - & 4.7 & $\mathrm{~V}_{\mathrm{c}}^{(2,14)}$ & 2.294 & 0.102 & 0.0975 \\
$\mathbf{7 5 5 8}$ & 4.1121 & - & 4.4 & $\mathrm{~V}^{(2,3,6,14)}$ & 2.290 & 0.110 & 0.0904 \\
$\mathbf{8 7 6 1}$ & 2.6386 & - & 3.7 & $\mathrm{~V}^{(2,9,14)}$ & 2.242 & 0.096 & 0.0639 \\
$\mathbf{9 4 8 1}$ & 3.0519 & - & 4.7 & $\mathrm{~V}^{(2,14,17)}$ & 2.287 & 0.139 & 0.036 \\
$\mathbf{9 5 3 1}$ & 2.5003 & - & 4.2 & $\mathrm{~V}^{(2,7,17,14)}$ & 2.235 & 0.136 & 0.0957 \\
10666 & 3.4947 & $3-$ & 4.0 & $\mathrm{~V}^{(2,11,14)}$ & 2.222 & 0.110 & 0.0934 \\
\hline & & & & & &
\end{tabular}

Notes. In bold, we flag asteroids for which we determined or confirmed periods in this article.

References. ${ }^{(1)}$ Neese (2010), ${ }^{(2)}$ Hasselmann et al. (2012), ${ }^{(3)}$ Wisniewski (1991), ${ }^{(4)}$ Florczak et al. (2002), ${ }^{(5)}$ Oszkiewicz et al. (2019), ${ }^{(6)}$ Moskovitz et al. (2008b), ${ }^{(7)}$ Solontoi et al. (2012), ${ }^{(8)}$ Alvarez-Candal et al. (2006), ${ }^{(9)}$ Sanctis et al. (2011), ${ }^{(10)}$ Fulvio et al. (2016), ${ }^{(11)} \mathrm{Hardersen}$ et al. (2018), ${ }^{(12)}$ Hardersen et al. (2014b), ${ }^{(13)}$ Licandro et al. (2017), ${ }^{(14)}$ Carvano et al. $(2010),{ }^{(15)}$ Bus \& Binzel $(2002),{ }^{(16)}$ DeMeo et al. (2009), ${ }^{(17)}$ Moskovitz et al. (2010), ${ }^{(18)}$ Popescu et al. (2018), ${ }^{(19)}$ Lazzaro et al. (2004), ${ }^{(20)}$ Solontoi et al. (2012). 
Table A.3. continued.

\begin{tabular}{rccrlccc}
\hline \hline $\begin{array}{r}\text { Asteroid } \\
\text { No. }\end{array}$ & $\begin{array}{c}\text { Synodic period } \\
\text { (LCDB) }(\mathrm{h})\end{array}$ & $\begin{array}{r}\text { U-code } \\
(\mathrm{LCDB})\end{array}$ & $\begin{array}{r}\text { Diameter } \\
(\mathrm{km})\end{array}$ & $\begin{array}{l}\text { Class, } \\
\text { references }\end{array}$ & $\begin{array}{c}a \\
(\mathrm{au})\end{array}$ & $e$ & $\sin (i)$ \\
\hline 14322 & 7.462 & 3 & 3.5 & $\mathrm{~V}^{(2,7,14)}$ & 2.234 & 0.106 & 0.1283 \\
15882 & 5.05 & 2 & 3.8 & $\mathrm{~V}_{\mathrm{c}}^{(2,14)}$ & 2.297 & 0.175 & 0.0419 \\
16416 & 36.506 & 2 & 3.4 & $\mathrm{~V}^{(2,14,17)}$ & 2.196 & 0.107 & 0.1079 \\
17028 & 12.80411 & 2 & 2.0 & $\mathrm{~V}_{\mathrm{c}}^{(2,14)}$ & 2.226 & 0.152 & 0.0758 \\
$\mathbf{2 2 1 1 3}$ & 6.7426 & - & 2.9 & $\mathrm{~V}_{\mathrm{c}}^{(2,14)}$ & 2.239 & 0.121 & 0.0958 \\
23615 & 368. & 2 & 3.5 & $\mathrm{~V}_{\mathrm{c}}^{(2,14)}$ & 1.961 & 0.114 & 0.3890 \\
26433 & 7.933 & 2 & 2.6 & $\mathrm{~V}_{\mathrm{c}}^{(2,14,18)}$ & 2.264 & 0.110 & 0.1252 \\
32008 & 3.0171 & 3 & 3.1 & $\mathrm{~V}_{\mathrm{c}}^{(2,14)}$ & 2.192 & 0.144 & 0.1221 \\
37142 & 47.068 & 2 & 1.5 & $\mathrm{~V}_{\mathrm{c}}^{(2,14)}$ & 2.229 & 0.122 & 0.1147 \\
48635 & 28.106 & 2 & 2.4 & $\mathrm{~V}_{\mathrm{c}}^{(2,14)}$ & 2.266 & 0.107 & 0.1034 \\
70069 & 6.252 & 2 & 1.7 & $\mathrm{~V}_{\mathrm{c}}^{(2,14)}$ & 2.231 & 0.105 & 0.1073 \\
84975 & 22.711 & 2 & 1.4 & $\mathrm{~V}_{\mathrm{c}}^{(2,14)}$ & 2.293 & 0.126 & 0.1149 \\
187060 & 8.125 & 2 & 1.0 & $\mathrm{~V}_{\mathrm{c}}^{(2,14)}$ & 2.241 & 0.097 & 0.1318 \\
\hline
\end{tabular}

Table A.4. As in Table A.2, but for the low inclination population.

\begin{tabular}{|c|c|c|c|c|c|c|c|}
\hline $\begin{array}{r}\text { Asteroid } \\
\text { No. }\end{array}$ & $\begin{array}{l}\text { Synodic period } \\
\quad \text { (LCDB) }(\mathrm{h})\end{array}$ & $\begin{array}{l}\text { U-code } \\
\text { (LCDB) }\end{array}$ & $\begin{array}{r}\text { Diameter } \\
(\mathrm{km}) \\
\end{array}$ & $\begin{array}{l}\text { Class, } \\
\text { references }\end{array}$ & $\begin{array}{c}a \\
(\mathrm{au})\end{array}$ & $e$ & $\sin (i)$ \\
\hline 1914 & 6.331 & 2 & 9.6 & $\mathrm{~V}^{(2,5,12)}$ & 2.406 & 0.139 & 0.0848 \\
\hline 2247 & 4.4974 & - & 4.4 & $\mathrm{~V}^{(2,12,16)}$ & 2.449 & 0.090 & 0.1017 \\
\hline 2442 & 11.453 & 2 & 8.3 & $\mathrm{~V}^{(1,2,12,14)}, \mathbf{J}^{(8,18)}$ & 2.388 & 0.097 & 0.0944 \\
\hline 2566 & 4.451 & 3 & 7.8 & $\mathrm{~V}^{(1,13,14,15)}$ & 2.450 & 0.104 & 0.0771 \\
\hline 2653 & 5.5228 & 3 & 9.9 & $\mathbf{V}^{(1,13,15)}$ & 2.444 & 0.114 & 0.0888 \\
\hline 2704 & 2.6382 & 3 & 5.2 & $\mathrm{~V}^{(1,2,12,13)}$ & 2.385 & 0.117 & 0.0889 \\
\hline 2763 & 7.8 & 3 & 7.5 & $\mathrm{~V}^{(1,3,9,11,13,15,17)}, \mathrm{S}^{(19)}$ & 2.404 & 0.179 & 0.0756 \\
\hline 2823 & 2.6262 & - & 5.4 & $\mathrm{~V}^{(2,4,12,15)}$ & 2.326 & 0.175 & 0.0726 \\
\hline 3331 & 7.5888 & - & 4.4 & $\mathrm{~V}_{\mathrm{c}}^{(2,10,11,12)}$ & 2.418 & 0.112 & 0.0624 \\
\hline 3849 & 2.778 & 2 & 5.8 & $\mathrm{~V}^{(1,2,6,12,13)}$ & 2.474 & 0.065 & 0.0940 \\
\hline 3867 & 28.035 & 2 & 5.4 & $\mathrm{~V}_{\mathrm{c}}^{(2,12)}$ & 2.351 & 0.136 & 0.0954 \\
\hline 4188 & 2.58 & $3-$ & 6.6 & $\mathrm{~V}^{(1,2,12,13,14,15)}$ & 2.335 & 0.111 & 0.0986 \\
\hline 4796 & 3.5086 & 3 & 5.7 & $\mathrm{~V}^{(1,2,7,12,13,15)}$ & 2.355 & 0.141 & 0.0538 \\
\hline 7798 & 3.8724 & - & 5.0 & $\mathrm{~V}_{\mathrm{c}}^{(2,12)}$ & 2.479 & 0.131 & 0.0800 \\
\hline 7899 & 85.658 & 2 & 3.9 & $\mathrm{~V}_{\mathrm{c}}^{(2,12)}$ & 2.343 & 0.114 & 0.0937 \\
\hline 8645 & 7.616 & $3-$ & 5.1 & $\mathrm{~V}^{(2,7,12)}$ & 2.431 & 0.041 & 0.0885 \\
\hline 9223 & 3.758 & 2 & 4.5 & $\mathrm{~V}^{(2,6,12)}$ & 2.301 & 0.080 & 0.0699 \\
\hline 11149 & 12.85 & $3-$ & 3.5 & $\mathrm{~V}_{\mathrm{c}}^{(2,12)}$ & 2.442 & 0.079 & 0.1035 \\
\hline 15481 & 5.01 & 2 & 4.7 & $\mathrm{~V}_{\mathrm{c}}^{(2,12)}$ & 2.383 & 0.107 & 0.0784 \\
\hline 15630 & 8.064 & 2 & 3.6 & $\mathrm{~V}^{(2,6,12,17)}$ & 2.326 & 0.146 & 0.0818 \\
\hline 27192 & 5.5741 & - & 3.2 & $\mathrm{~V}_{\mathrm{c}}^{(2,12)}$ & 2.323 & 0.119 & 0.097 \\
\hline 29337 & 4.5096 & 3 & 4.2 & $\mathrm{~V}_{\mathrm{c}}^{(2,12)}$ & 2.468 & 0.189 & 0.1025 \\
\hline 47417 & 11.71 & $2-$ & 4.6 & $\mathrm{~V}_{\mathrm{c}}^{(2,12)}$ & 2.400 & 0.090 & 0.0990 \\
\hline 56570 & 3.22 & 2 & 2.4 & $\mathrm{~V}^{(2,11,12)}$ & 2.378 & 0.096 & 0.0721 \\
\hline 82125 & 2.554 & 2 & 1.8 & $\mathrm{~V}_{\mathrm{c}}^{(2,12)}$ & 2.414 & 0.092 & 0.0976 \\
\hline 118567 & 6.741 & 2 & 1.3 & $\mathrm{~V}_{\mathrm{c}}^{(2,12)}$ & 2.318 & 0.093 & 0.1026 \\
\hline 151036 & 4.974 & 2 & 1.9 & $\mathrm{~V}_{\mathrm{c}}^{(2,12)}$ & 2.448 & 0.124 & 0.0957 \\
\hline
\end{tabular}

Notes. In bold we flag asteroids for which we determined or confirmed periods in this article.

References. ${ }^{(1)}$ Neese (2010), ${ }^{(2)}$ Hasselmann et al. (2012), ${ }^{(3)}$ Duffard et al. (2004), ${ }^{(4)}$ Moskovitz et al. (2008b), ${ }^{(5)}$ Alvarez-Candal et al. (2006), ${ }^{(6)}$ Hardersen et al. (2018), ${ }^{(7)}$ Hasegawa et al. (2014), ${ }^{(8)} \mathrm{Xu}$ et al. $(1995),{ }^{(9)}$ Spahr et al. (1997), ${ }^{(10)}$ Roig \& Gil-Hutton (2006), ${ }^{(11)}$ Licandro et al. (2017), ${ }^{(12)}$ Carvano et al. (2010), ${ }^{(13)}$ Bus \& Binzel (2002), ${ }^{(14)}$ DeMeo et al. (2009), ${ }^{(15)}$ Moskovitz et al. (2010), ${ }^{(16)}$ Hicks et al. (2014), ${ }^{(17)}$ Popescu et al. (2018), ${ }^{(18)}$ Binzel \& Xu (1993), ${ }^{(19)}$ Erasmus et al. (2019). 
Table A.5. As in Table A.2, but for the inner other population.

\begin{tabular}{|c|c|c|c|c|c|c|c|}
\hline $\begin{array}{r}\text { Asteroid } \\
\text { No. }\end{array}$ & $\begin{array}{c}\text { Synodic period } \\
\quad \text { (LCDB) (h) }\end{array}$ & $\begin{array}{r}\text { U-code } \\
\text { (LCDB) }\end{array}$ & $\begin{array}{r}\text { Diameter } \\
(\mathrm{km}) \\
\end{array}$ & $\begin{array}{l}\text { Class, } \\
\text { references }\end{array}$ & $\begin{array}{c}a \\
(\mathrm{au})\end{array}$ & $e$ & $\sin (i)$ \\
\hline 854 & 37.56 & 3 & 7.8 & $\mathrm{~V}^{(2,3,12)}$ & 2.368 & 0.164 & 0.1124 \\
\hline 2432 & 3.206 & $3-$ & 7.4 & $\mathrm{~V}_{\mathrm{c}}^{(2,12)}$ & 2.352 & 0.129 & 0.1158 \\
\hline 2851 & 5.414 & $3-$ & 8.8 & $\mathrm{~V}^{(1,13,14,15)}$ & 2.478 & 0.123 & 0.1348 \\
\hline 3536 & 5.79 & 3 & 3.2 & $\mathrm{~V}^{(1,22,26,31,35)}$ & 2.343 & 0.077 & 0.1156 \\
\hline 3900 & 5.324 & 3 & 5.0 & $\mathbf{V}^{(1,7,10,11,13,17)}$ & 2.371 & 0.113 & 0.1224 \\
\hline 4383 & 3.4069 & 3 & 6.5 & $\mathrm{~V}^{(2,7,9,12)}$ & 2.425 & 0.055 & 0.1095 \\
\hline 5150 & 5.1953 & 3 & 5.4 & $\mathrm{~V}_{\mathrm{c}}^{(2,11,12,17)}$ & 2.477 & 0.138 & 0.1076 \\
\hline 5524 & 8.416 & 3 & 19.9 & $\mathrm{~V}_{\mathrm{c}}^{(2,11,12,17)}$ & 2.366 & 0.059 & 0.1194 \\
\hline 5599 & 3.6195 & 3 & 6.4 & $\mathrm{~V}^{(2,12,16)}$ & 2.419 & 0.155 & 0.1264 \\
\hline 5875 & 5.551 & 3 & 7.5 & $\mathrm{~V}^{(2,6,11,12,17)}$ & 2.379 & 0.067 & 0.1246 \\
\hline 6680 & 6.722 & 3 & 4.5 & $\mathrm{~V}_{\mathrm{c}}^{(2,12)}$ & 2.322 & 0.095 & 0.1408 \\
\hline 6976 & 4.025 & 3 & 5.5 & $\mathrm{~V}_{\mathrm{c}}^{(2,12)}$ & 2.333 & 0.119 & 0.1303 \\
\hline 7484 & 6.237 & 2 & 4.0 & $\mathrm{~V}_{\mathrm{c}}^{(2,12)}$ & 2.405 & 0.071 & 0.1114 \\
\hline 9368 & 2.9183 & 3 & 4.3 & $\mathrm{~V}^{(2,5,12)}$ & 2.310 & 0.163 & 0.1289 \\
\hline 9652 & 4.385 & 2 & 6.0 & $\mathrm{~V}_{\mathrm{c}}^{(2,12)}$ & 2.418 & 0.197 & 0.1196 \\
\hline 18641 & 5.2461 & 3 & 3.7 & $\mathrm{~V}_{\mathrm{c}}^{(2,12)}$ & 2.357 & 0.097 & 0.1344 \\
\hline 25327 & 6.716 & 3 & 4.4 & $\mathrm{~V}^{(4)}$ & 2.434 & 0.186 & 0.2187 \\
\hline 25542 & 10.55227 & 2 & 2.7 & $\mathrm{~V}_{\mathrm{c}}^{(2,12)}$ & 2.443 & 0.116 & 0.1224 \\
\hline 35062 & 19.08 & 2 & 3.2 & $\mathrm{~V}^{(2,4,11,12,17)}$ & 2.370 & 0.285 & 0.1755 \\
\hline 42979 & 3.498 & 2 & 2.0 & $\mathrm{~V}_{\mathrm{c}}^{(2,12)}$ & 2.400 & 0.110 & 0.1198 \\
\hline 53533 & 5.674 & 2 & 2.4 & $\mathrm{~V}_{\mathrm{c}}^{(2,12)}$ & 2.419 & 0.137 & 0.1082 \\
\hline 54584 & 2.92 & $3-$ & 3.2 & $\mathrm{~V}_{\mathrm{c}}^{(2,12)}$ & 2.342 & 0.123 & 0.1431 \\
\hline
\end{tabular}

References. ${ }^{(1)}$ Neese (2010), ${ }^{(2)}$ Hasselmann et al. (2012), ${ }^{(3)}$ Birlan et al. (2011), ${ }^{(4)}$ Solontoi et al. (2012), ${ }^{(5)}$ Hardersen et al. (2018), ${ }^{(6)}$ Hardersen et al. (2015), ${ }^{(7)}$ Hasegawa et al. (2014), ${ }^{(8)} \mathrm{Xu}$ et al. (1995), ${ }^{(9)}$ Pajuelo et al. (2018), ${ }^{(10)}$ Spahr et al. (1997), ${ }^{(11)}$ Licandro et al. (2017), ${ }^{(12)}$ Carvano et al. (2010), ${ }^{(13)}$ Bus \& Binzel (2002), ${ }^{(14)}$ DeMeo et al. (2009), ${ }^{(15)}$ Moskovitz et al. (2010), ${ }^{(16)}$ Hicks et al. (2014), ${ }^{(17)}$ Popescu et al. (2018). 
Table A.6. As in Table A.2, but for the NEA population.

\begin{tabular}{rccclccc}
\hline \hline $\begin{array}{r}\text { Asteroid } \\
\text { No. }\end{array}$ & $\begin{array}{c}\text { Synodic period } \\
\text { (LCDB) }(\mathrm{h})\end{array}$ & $\begin{array}{r}\text { U-code } \\
(\mathrm{LCDB})\end{array}$ & $\begin{array}{r}\text { Diameter } \\
(\mathrm{km})\end{array}$ & $\begin{array}{l}\text { Class, } \\
\text { references }\end{array}$ & $\begin{array}{c}a \\
(\mathrm{au})\end{array}$ & $e$ & $\sin (i)$ \\
\hline 1981 & 5.22 & 3 & 3.4 & $\mathrm{~V}^{(6)}$ & 0.650 & 1.777 & 0.6400 \\
3361 & 3.532 & 3 & 0.3 & $\mathrm{~V}^{(3,17)}$ & 0.323 & 1.210 & 0.0470 \\
3551 & 4.93 & 2 & 0.9 & $\mathrm{~V}^{(1,11)}$ & 0.487 & 2.093 & 0.1650 \\
3908 & 4.42601 & 3 & 1.0 & $\mathrm{~V}^{(1,7,15)}$ & 0.459 & 1.927 & 0.0380 \\
4055 & 7.475 & 3 & 2.5 & $\mathrm{~V}^{(1,2,5,7,8,10,11,13,14,16,18,19)}$ & 0.326 & 1.820 & 0.3950 \\
5604 & 5.3375 & 3 & 0.6 & $\mathrm{~V}^{(1,5,7,13)}$ & 0.405 & 0.927 & 0.0840 \\
6611 & 2.5568 & 3 & 0.8 & $\mathrm{~V}^{(1,12)}$ & 0.485 & 1.696 & 0.1500 \\
7889 & 2.741 & 3 & 1.7 & $\mathrm{~V}^{(4,7)}, \mathrm{R}^{(20)}$ & 1.261 & 0.346 & 0.6000 \\
52750 & 26.43 & $2+$ & 1.1 & $\mathrm{~V}^{(4,5)}$ & 0.525 & 1.427 & 0.1940 \\
55532 & 46.08 & 2 & 1.3 & $\mathrm{~V}^{(18)}, \mathrm{Sk}^{(7)}$ & 1.794 & 0.696 & 0.623 \\
137052 & 9.007 & $3-$ & 0.9 & $\mathrm{~V}^{(1,12)}$ & 0.810 & 1.248 & 0.2570 \\
137199 & 2.767 & 3 & 1.2 & $\mathrm{~V}^{(21)}, \mathrm{Q}^{(18)}$ & 1.457 & 0.293 & 0.285 \\
137924 & 10.57 & $3-$ & 1.0 & $\mathrm{~V}^{(5)}$ & 0.895 & 0.876 & 0.4340 \\
152756 & 2.845 & 3 & 0.5 & $\mathrm{~V}^{(18)}, \mathrm{S}^{(6)}$ & 1.451 & 0.415 & 0.263 \\
163249 & 3.7663 & 3 & 0.6 & $\mathrm{~V}^{(18)}$ & 1.344 & 0.335 & 0.121 \\
164121 & 2.343 & 3 & 1.7 & $\mathrm{~V}^{(5,19)}$ & 1.11 & 0.292 & 0.695 \\
297418 & 6.314 & 3 & 0.4 & $\mathrm{~V}^{(5)}$ & 0.467 & 0.811 & 0.1800 \\
389694 & 5.24 & $2-$ & 0.5 & $\mathrm{~V}^{(18)}$ & 1.545 & 0.492 & 0.327 \\
477762 & 15.04 & $2-$ & 0.3 & $\mathrm{QV}^{(18)}$ & 2.06 & 0.495 & 0.205 \\
$1996 \mathrm{JA} 1$ & 5.227 & 3 & 0.2 & $\mathrm{~V}^{(9)}$ & 0.701 & 2.567 & 0.3680 \\
1997 GL3 & 7.572 & 2 & 0.3 & $\mathrm{~V}^{(1,12)}$ & 0.782 & 2.276 & 0.1160 \\
\hline
\end{tabular}

References. ${ }^{(1)}$ Neese (2010), ${ }^{(2)}$ Hasselmann et al. (2012), ${ }^{(3)}$ Wisniewski (1991), ${ }^{(4)}$ Duffard et al. (2004), ${ }^{(5)}$ Thomas et al. (2014), ${ }^{(6)}$ Binzel et al. (2001), ${ }^{(7)}$ Binzel et al. (2004), ${ }^{(8)}$ Hardersen et al. (2018), ${ }^{(9)}$ Spahr et al. $(1997),{ }^{(10)}$ Carvano et al. (2010), ${ }^{(11)}$ Binzel et al. (1989), ${ }^{(12)}$ Bus \& Binzel (2002), ${ }^{(13)}$ DeMeo et al. (2009), ${ }^{(14)}$ Hicks et al. (2014), ${ }^{(15)}$ Popescu et al. (2018), ${ }^{(16)}$ Lazzaro et al. (2004), ${ }^{(17)}$ Hasegawa et al. (2018), ${ }^{(18)}$ Lin et al. (2018), ${ }^{(19)}$ Sanchez et al. (2013), ${ }^{(20)}$ Dandy et al. (2003), ${ }^{(21)}$ Carry et al. (2016).

Table A.7. As in Table A.2, but for the Middle/Outer Main Belt population.

\begin{tabular}{rccrlrcr}
\hline \hline $\begin{array}{r}\text { Asteroid } \\
\text { No. }\end{array}$ & $\begin{array}{c}\text { Synodic period } \\
\text { (LCDB) }(\mathrm{h})\end{array}$ & $\begin{array}{c}\text { U-code } \\
(\mathrm{LCDB})\end{array}$ & $\begin{array}{r}\text { Diameter } \\
(\mathrm{km})\end{array}$ & $\begin{array}{l}\text { Class, } \\
\text { references }\end{array}$ & $\begin{array}{c}a \\
(\mathrm{au})\end{array}$ & $e$ & $\sin (i)$ \\
\hline 1459 & 4.678 & 3 & 29.2 & $\mathrm{~V}^{(3,5,8)}, \mathrm{Vw}^{(1,7)}$ & 3.150 & 0.213 & 0.2681 \\
\hline
\end{tabular}

References. ${ }^{(1)}$ Neese (2010), ${ }^{(3)}$ Solontoi et al. (2012), ${ }^{(5)}$ Lazzaro et al. (2000), ${ }^{(7)}$ DeMeo et al. (2009), ${ }^{(8)}$ Lazzaro et al. (2004). 

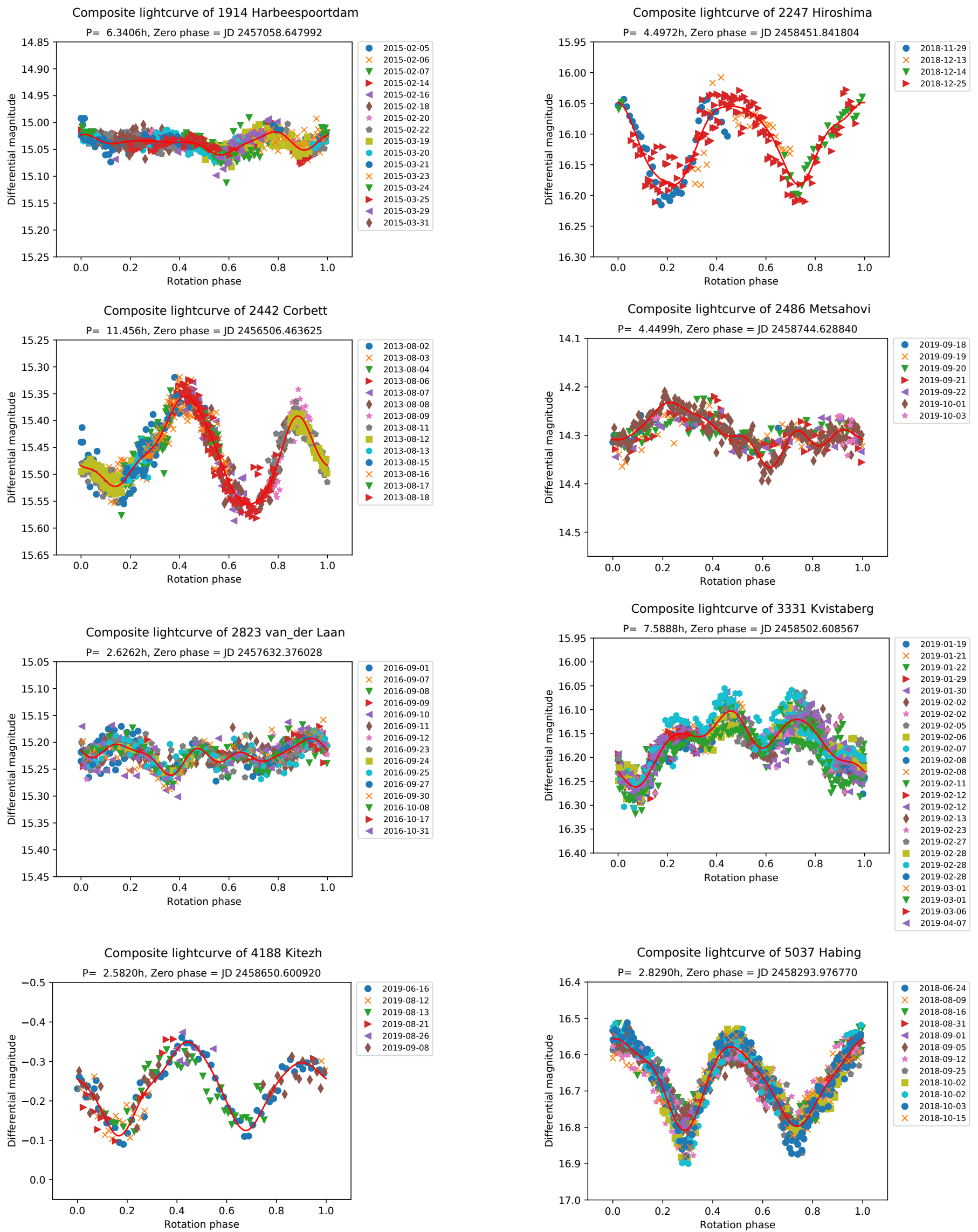

Fig. A.1. Composite light curves of the observed V-type asteroids (as in Table 4). 
D. Oszkiewicz et al.: Spin rates of V-types
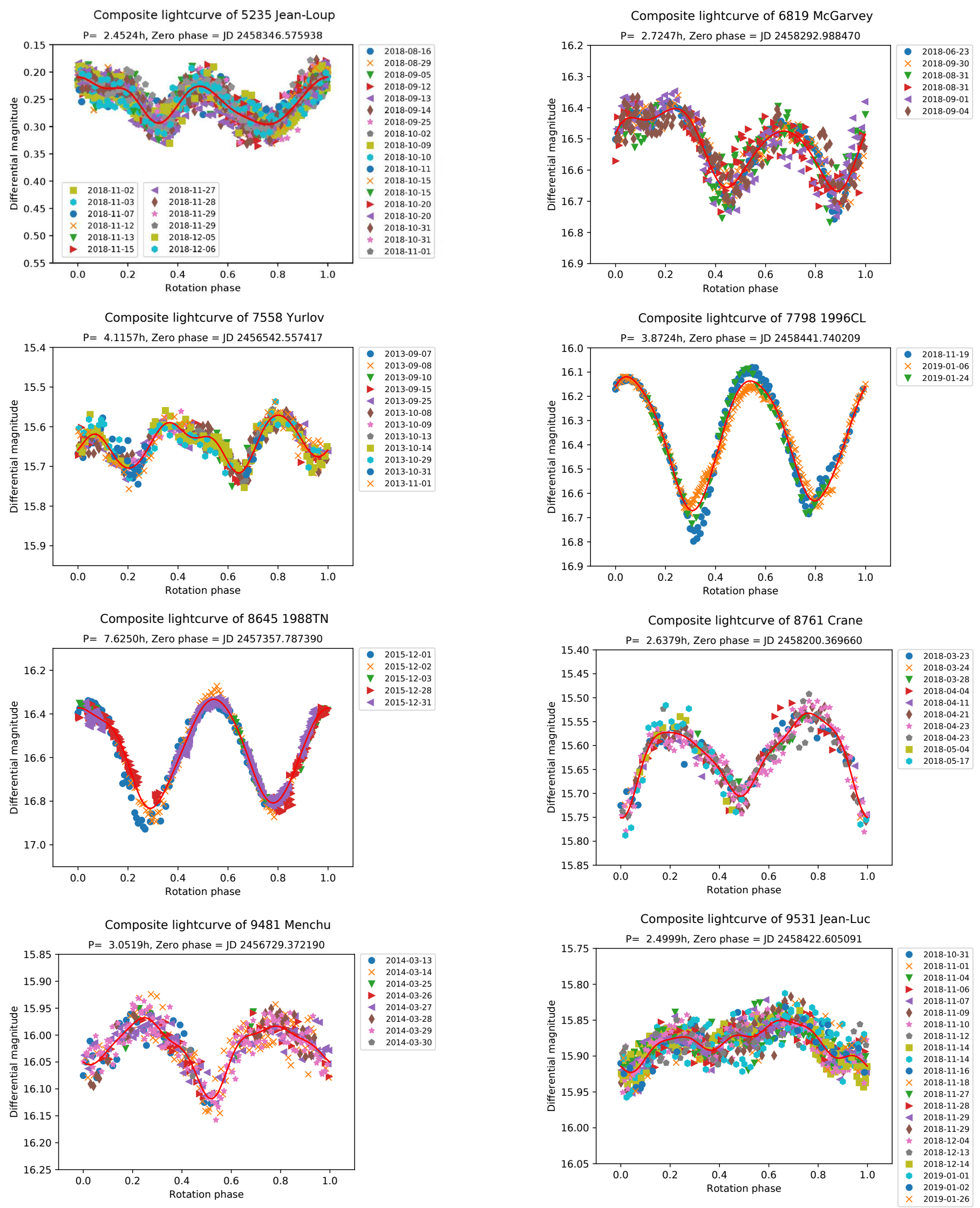

Fig. A.1. continued. 
A\&A 643, A117 (2020)
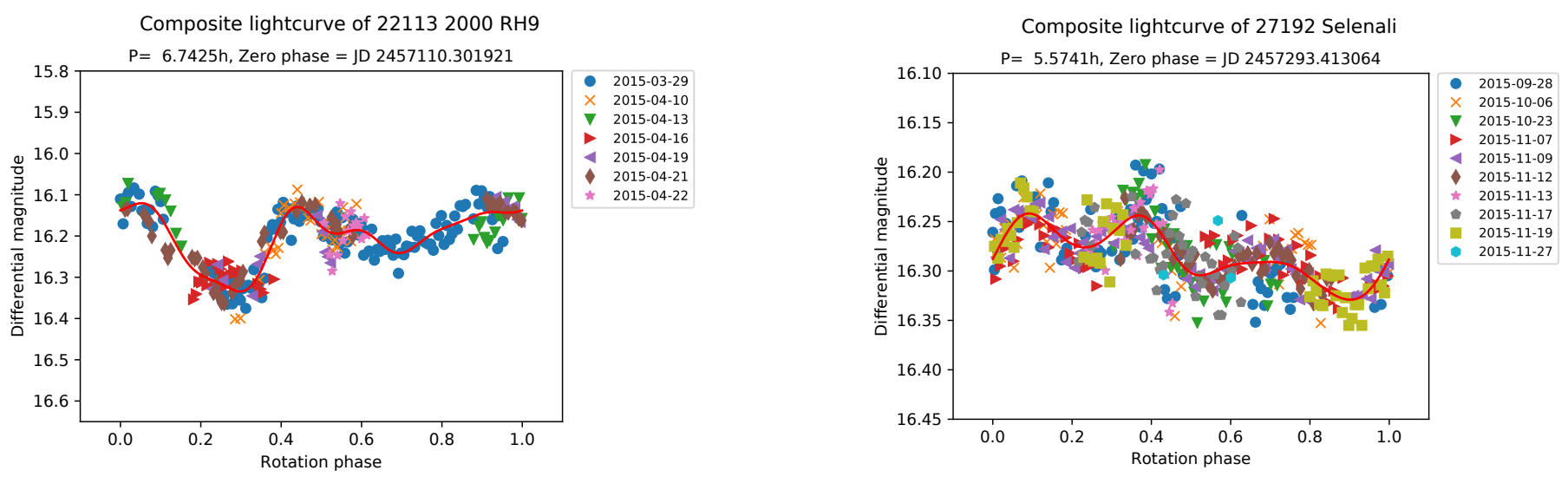

Fig. A.1. continued. 